\title{
Tuning Ca-Al-based catalysts composition to isomerize or epimerize glucose and other sugars
}

Received 00th January 20xx, Accepted 00th January 20xx DOI: $10.1039 / \times 0 \times x 00000 x$

\author{
Maria Ventura, ${ }^{* a}$ Juan A. Cecilia, ${ }^{\mathrm{b}}$ Enrique Rodríguez-Castellón, ${ }^{\mathrm{b}}$ and Marcelo E. Domine*a
}

One of the key reactions to achieve good productivity on the transformations of the cellulose-derived from biomass feedstocks is the isomerization of glucose to fructose, being this latest the platform molecule for obtaining other important derivatives. In this work, Ca-Al containing catalysts based on hydrotalcite-type derived materials were used to perform the selective isomerization of glucose to fructose, and the selective epimerization of glucose to mannose, using water as solvent under mild reaction conditions. The catalysts showed high activity (conversion $=51-87 \%$ ), and excellent selectivity $(63-88 \%$ ) towards fructose, compared with the current industrial process based on the glucose transformation via biocatalysis. It was also possible to modulate the selectivity towards fructose or mannose by tuning the amount of basic sites of the catalysts and its composition. The combination of basic and acid sites present in the Ca-Al-based catalysts plays a key role in the reaction, fact that is discussed in the text together with other important operational parameters. The stability and recyclability of the catalysts were tested detecting only a small activity loss after 5 consecutive runs. The synthesis of the catalysts and their characterization is also discussed since they are one of the few cases found in the literature of this kind of hydrotalcite-type materials with such a high level of $\mathrm{Ca}$ incorporation. Some green metrics, such as E-factor have been calculated to evaluate our system as an environmental friendly process.

\section{Introduction}

Fructose is a well-known sweetener used as an alternative to conventional table sugar (sucrose) as it is 1.8-2.0 fold sweeter than sucrose. ${ }^{1}$ It has also emerged as a key starting compound in biorefineries for the synthesis of value-added chemicals such as 5-hydroxymethylfurfural, 2,5-furandicarboxylic acid, levulinic acid or lactic acid, chemicals that can replace those synthetized from fossil feedstocks. ${ }^{2,3}$ Currently, fructose is industrially produced by the use of a biocatalytic process, employing xylose isomerase as enzyme. At the end of this process and after several HPLC enrichments the so-called high fructose syrup is attained. ${ }^{4}$ Although the enzymatic process is recognized as an effective method, it incurs high cost owing to the demand for purified feeding material, being biomass feedstock not suitable for this process; narrow operating conditions $(\mathrm{pH}$ and temperature); and irreversible deactivation of spent enzymes. ${ }^{5}$ Glucose synthetized from green resources, such as lignocellulosic biomass, represents an alternative to the classical feedstock; therefore, more emphasis should be placed on developing new systems, able to work with biomass derived substrates, based on more efficient and selective processes as well as more resistant to the reaction medium. In comparison,

\footnotetext{
a. Instituto de Tecnología Química, ITQ (UPV-CSIC). Universitat Politècnica de València. Consejo Superior de Investigaciones Científicas. Avda. Los Naranjos S/N, 46022, Valencia, Spain.

b. Departamento de Química Inorgánica, Cristalografía y Mineralogía (Unidad Asociada al ICP-CSIC), Facultad de Ciencias, Universidad de Málaga, Campus de Teatinos, 29071 Málaga, Spain.

Electronic Supplementary Information (ESI) available: [details of any supplementary information available should be included here]. See DOI: 10.1039/x0xx00000x
}

chemical catalysts show the advantages of significantly shorter reaction time and greater endurance to impurities. Glucose isomerization can be catalysed by Lewis acids (i.e., electron pair acceptor such as $\mathrm{Sn}^{4+}$ and $\mathrm{Al}^{3+}$ ) via intramolecular hydride shift from $\mathrm{C}-2$ to $\mathrm{C}-1,6,7$ by $\mathrm{Br} \varnothing$ nsted (i.e., $\mathrm{OH}^{-}$) or Lewis bases via hydrogen transfer from $\mathrm{O}^{-2}$ to $\mathrm{O}^{-1}, 8,9$ or by a combination of both functionalities. ${ }^{10}$ Homogeneous catalysts provide good catalytic performance, ${ }^{11}$ but often raise concerns about equipment corrosion as well as downstream product separation and solvent treatment, which has promoted the development of heterogeneous solid catalysts for biomass conversion. ${ }^{12}$ The use of fructose as intermediate for the production of valuable furan derivatives and platform chemicals (Scheme 1) has spurred the quest for sustainable and cheap catalysts to replace the enzymatic systems. ${ }^{13,14}$ As it is shown in Scheme 1 , the isomerization of glucose to fructose is accompanied by a variety of by-products. Consequently, highly selective, efficient and inexpensive catalysts are essential to the control of undesirable reactions improving the reaction kinetics and to develop a system economically more favourable.

Mg-Al mixed oxides have been used as effective catalysts for this process reporting good fructose selectivity at glucose conversion below $40 \%{ }^{12}$ These metallic mixed oxides derived from hydrotalcites, which are layered double hydroxides consisting of a mixture of $\mathrm{M}^{\prime \prime}$ and $\mathrm{M}^{\prime \prime \prime}$, have also exhibited good catalytic performance (HT-Mg/Al) with a high selectivity (83\%) at high glucose conversion $(61 \%)$ by using ethanol:water mixture as solvent. ${ }^{15}$ Many other heterogeneous catalysts have been developed for this important process but none of them are comparable to enzymatic isomerization of glucose that 
selectively synthetizes fructose with an almost quantitative yield (ca. 50\%).

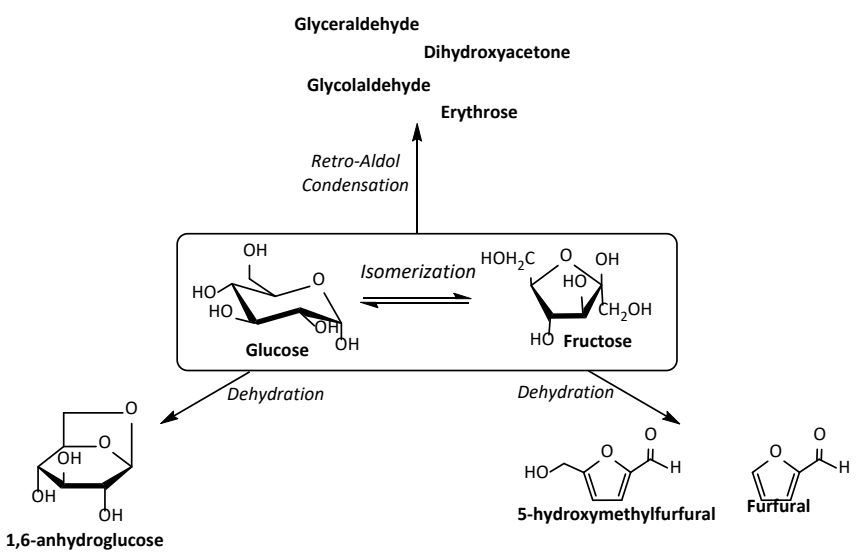

Scheme 1. Isomerization of Glucose to Fructose and side reactions taking place during the process.

Glucose epimerization to mannose arises in nearly every investigation of glucose conversion and competes with the isomerization reaction. Just a few examples have been found in the literature about the selective production of mannose catalyzed by heterogeneous catalysts, both acid and basic systems. Sn-Beta zeolites were used to react glucose in methanol solvent where mannose was produced selectively via a mechanism known as the Bilik reaction. ${ }^{16}$ Although other homogeneous and heterogeneous catalysts able to catalyze the epimerization of glucose to mannose have been reported, ${ }^{17,18}$ basic catalysts have not been explored so far because of the poor selectivity towards mannose, being the isomerization to fructose always favoured. To the best of our knowledge, metallic mixed oxides derived from hydrotalcite-type precursors have not been explored to mediate this reaction.

In the present work, metallic mixed oxides containing Calcium and Aluminium were synthetized via hydrotalcite-type precursor structure, with a very high yield $(\geq 75 \%)$, this being one of the first examples of the synthesis of these materials minimizing or even avoiding the presence of $\mathrm{CaCO}_{3} \cdot{ }^{19,20}$ Then, the Ca-Al-based materials were used to isomerize or epimerize glucose under mild reaction conditions. Catalysts were first fully characterized, followed by a catalysts screening study to modulate the selectivity of the reaction towards fructose or mannose. The effects of temperature, pressure as well as catalysts composition were investigated. Some insights of the mechanisms were also explored by following the products formation on the time reaction course. The application scope of the Ca-Al-based materials was demonstrated, catalysts showing good performance in the isomerization of other sugars such as xylose or lactose.

\section{Results and discussion}

\section{HT-precursors and Ca-Al-based catalysts characterization:}

Main textural and physicochemical properties of $\mathrm{Ca}-\mathrm{Al}$ based materials with different $\mathrm{Ca} / \mathrm{Al}$ molar ratios synthesized by the co-precipitation method (see Experimental procedure section) and used in this work are summarized in Table 1. It is worth to noting that Ca-Al (0.8) sample possesses a surface area of 116 $\mathrm{m}^{2} / \mathrm{g}$, which is almost 20 folds of that of the other Ca-Al mixed oxides presented in this study. As Ca/Al molar ratios in solids surpass the value of 1 , surface areas dramatically decrease because of the presence of high amount of Ca. The same effect was observed for the pore volume that drastically decreases with the increased amount of $\mathrm{Ca}$.

Table 1. Main textural and physicochemical properties of Ca-Al mixed oxides.

\begin{tabular}{|c|c|c|c|c|}
\hline Sample & $\begin{array}{l}\mathrm{Ca} / \mathrm{Al} \\
\text { molar } \\
\text { ratio }^{\mathrm{a}}\end{array}$ & $\begin{array}{c}\text { Mixed } \\
\text { oxide } \\
\text { structure }\end{array}$ & $\begin{array}{c}\text { Surface } \\
\text { area } \\
\left(\mathrm{m}^{2} / g\right)^{b} \\
\end{array}$ & $\begin{array}{c}\text { Pore } \\
\text { volume } \\
\left(\mathrm{cm}^{3} / \mathrm{g}\right)^{\mathrm{b}}\end{array}$ \\
\hline Ca-Al (0.8) & $0.8 / 1$ & $\mathrm{Ca}_{0.8} \mathrm{AlO}_{2.3}$ & 116 & 0.420 \\
\hline Ca-Al (2.4) & $2.4 / 1$ & $\mathrm{Ca}_{2.4} \mathrm{AlO}_{3.9}$ & 6 & 0.029 \\
\hline Ca-Al (3.0) & $3.0 / 1$ & $\mathrm{Ca}_{3.0} \mathrm{AlO}_{4.5}$ & 5 & 0.032 \\
\hline Ca-Al (3.4) & $3.4 / 1$ & $\mathrm{Ca}_{3.4} \mathrm{AlO}_{4.9}$ & 7 & 0.041 \\
\hline
\end{tabular}

a Values obtained from ICP measurements. ${ }^{b}$ Values calculated from $\mathrm{N}_{2}$ adsorption isotherms (BET method).

The crystalline structures of the as synthetized HT-precursors and the Ca-Al mixed oxides (with different Ca:Al molar ratios) obtained after calcination were characterized by XRD measurements, and their XRD patterns are shown in Figure 1.
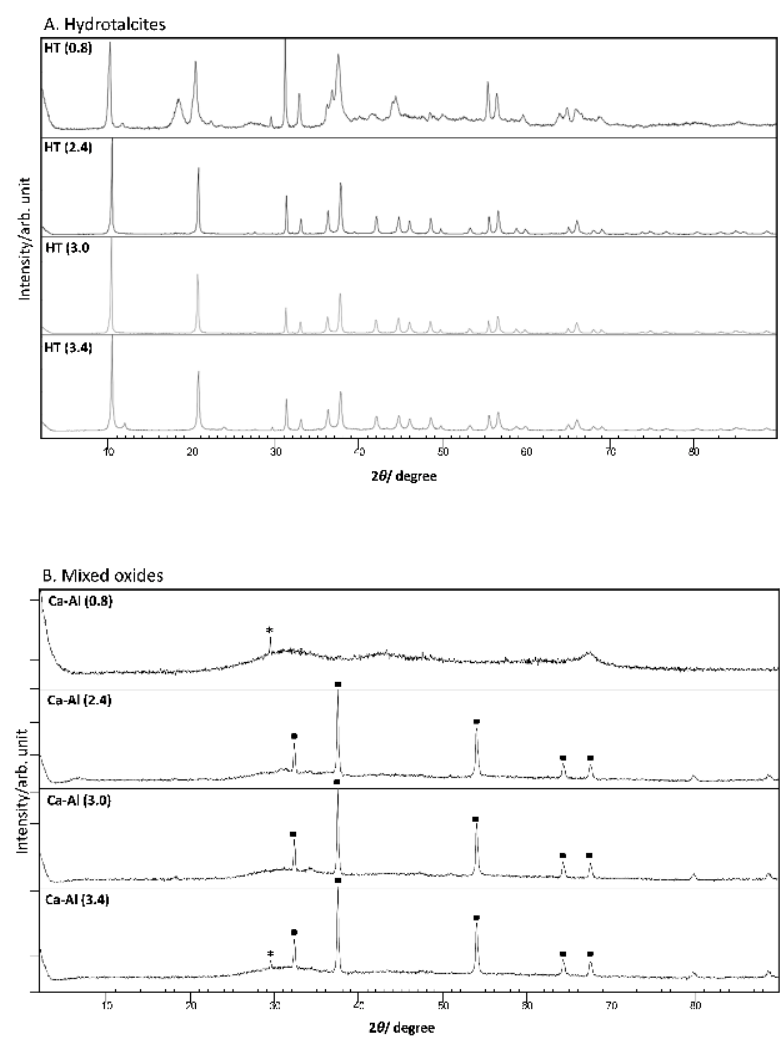

Figure 1. XRD patterns of A. HT-precursor materials; and B. CaAl mixed oxides obtained from the calcination of HT-precursors. Legends: stars: $\mathrm{CaCO}_{3}$; squares: $\mathrm{CaO}$. 
The patterns for HT-precursors series $(0.8 ; 2.4 ; 3.0$; and 3.4$)$ clearly confirm the layered structure of the materials, which is consistent with others hydrotalcite-type structures characterization. ${ }^{20,21}$ Three relatively narrow peaks appear at low $2 \theta$ values corresponding to the 003, 006 and 012 reflections. The interlayer distance can be calculated from the diffraction peak at $10.1-10.3^{\circ}$, which originates from the (003) plane of HT-precursors (Table 2).

Table 2. Interlayer distance calculated based on Bragg's law.

\begin{tabular}{|c|c|c|}
\hline Sample & $2 \theta$ & $d(\AA)$ \\
\hline HT (0.8) & 10.15 & 8.71 \\
\hline HT (2.4) & 10.32 & 8.56 \\
\hline HT (3.0) & 10.25 & 8.62 \\
\hline HT (3.4) & 10.34 & 8.56 \\
\hline
\end{tabular}

As it is shown in Table 2, the interlayer distance is higher to that observed for classical hydrotalcite comprising of a mixture of $\mathrm{Mg}: \mathrm{Al}\left(\approx 7.8 \AA\right.$ A),${ }^{22}$ because of the higher atomic radius of $\mathrm{Ca}$ ( 2.31 $\AA$ A) compared with the value of $\mathrm{Mg}(1.73 \AA)$. This fact increases the interlayer space making it bigger and increasing at the same time the crystallinity of the solid. No other phases were identify in the X-rays diffraction patterns; although HT (0.8) sample shows a pattern identified as a mixture of the HT structure and other non-identified phase. It is worth to highlight the absence of $\mathrm{CaCO}_{3}$, usually present in this kind of hydrotalcite-type materials. ${ }^{19,21}$ When the synthesis is performed without any control in the atmosphere is often observed the segregation of a $\mathrm{CaCO}_{3}$ phase instead of the formation of the desired HT-like phase. This fact is presumably attributed to the greater insolubility of $\mathrm{CaCO}_{3}$ formed by the reaction of calcium and $\mathrm{Na}_{2} \mathrm{CO}_{3}$, or ambient $\mathrm{CO}_{2}$, commonly used to introduce $\mathrm{CO}_{3}{ }^{2-}$ in the interlayer space of the hydrotalcite, ${ }^{23}$ and the incompatibility of the atomic radius of $\mathrm{Al}$ and $\mathrm{Ca}$ (1.43 versus $2.31 \AA ̊)$. The hydrotalcite-precursors synthesized here did not present practically any rest of $\mathrm{CaCO}_{3}$ (or its amount is very low), as it was demonstrated in the XRD pattern. After calcination the XRD patterns obtained for Ca-Al mixed oxides with intermediate $\mathrm{Ca} / \mathrm{Al}$ molar ratios (2.4 and 3.0) shows only pure $\mathrm{CaO}$ phase (Figure 1B). Calcination treatment of the samples was made at temperatures $\left(\begin{array}{lll}723 & \mathrm{~K}\end{array}\right)$ lower than the needed for the decomposition of $\mathrm{CaCO}_{3}(1023 \mathrm{~K})$ that further shows the absence of $\mathrm{CaCO}_{3}$ in the as-prepared $\mathrm{HT}$-precursors. In the cases of the highest [HT (3.4)] or lowest [HT (0.8)] molar ratios of $\mathrm{Ca} / \mathrm{Al}$ a bit proportion of $\mathrm{CaCO}_{3}$ was detected. For the $\mathrm{Ca}-\mathrm{Al}(0.8)$ mixed oxide, it was impossible to calculate the proportion of $\mathrm{CaCO}_{3}$ because of the very poor crystallinity of the sample. However, the high crystallinity shown for the solid having the highest $\mathrm{Ca} / \mathrm{Al}$ molar ratio [Ca-Al (3.4)], allowed to make an estimation of the value giving a $7.3 \%$ of $\mathrm{CaCO}_{3}$. Some authors postulated that for the HT-precursors made up by $\mathrm{Ca}$ and $\mathrm{Al}$, the metastable HT phase formed in these solids, can evolve to a one more stable identified as katoite. ${ }^{24}$ In our case, we could dismiss its formation by comparison of our XRD patterns with a reference pattern from the literature. ${ }^{25}$

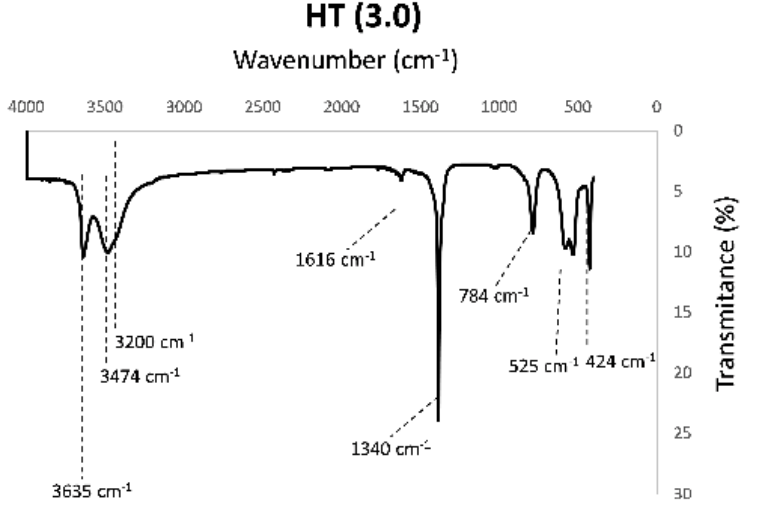

Figure 2. FT-IR spectrum of the HT (3.0) sample.

FT-IR measurements of the HT-precursors, before calcined, were investigated in order to know the composition of these new structures. Figure 2 shows the FT-IR spectrum of HT (3.0) sample. Only one spectrum is shown because of the similarity between all of them (Figure S1). The peak at $3635 \mathrm{~cm}^{-1}$ is assigned to the hydroxyl group vibration in the Ca-Al HT layer, and the shoulder signal at $3200 \mathrm{~cm}^{-1}$ corresponds to hydrogen bonding between interlayer water and $\mathrm{NO}_{3}{ }^{-}$in the $\mathrm{Ca}-\mathrm{Al}-\mathrm{NO}_{3}$ HT-type structure. The peak at $3474 \mathrm{~cm}^{-1}$ could be only attributed to the hydrogen bonding between $\mathrm{N}$ and $\mathrm{H}$, which confirms the absence of $\mathrm{CO}_{3}{ }^{2-}$. As is written in the experimental part, the synthesis of these hydrotalcite-type materials is done under inert atmosphere, to avoid the formation of $\mathrm{CaCO}_{3}$. Therefore, the presence of $\mathrm{CO}_{3}{ }^{2-}$ is highly minimized, but does not rule out the possibility that part of the $\mathrm{CO}_{2}$ present in the water of the nitrate solutions could participate in the reaction helping to create the layers of the hydrotalcite. In fact, the peak at $1340 \mathrm{~cm}^{-1}$ can be also attributed to the vibration of interlayer $\mathrm{CO}_{3}{ }^{2-}$. The feature at $1616 \mathrm{~cm}^{-1}$ represents the $\mathrm{H}_{2} \mathrm{O}$ bending of the interlayer water present in HT layers. ${ }^{26}$ Peaks below $800 \mathrm{~cm}$ ${ }^{1}$ could be attributed to the vibration of the metal-O bonds.

To elucidate and quantify the basic and acid sites present in each synthesized Ca-Al mixed oxide (derived from the calcination of HT-precursor), which may be a key factor in the isomerization/epimerization of glucose, TPD analysis were performed by using $\mathrm{CO}_{2}$ and $\mathrm{NH}_{3}$ as gaseous probe molecules for basic and acid sites, respectively. In the case of evaluation of the total number of $\mathrm{CO}_{2}$ molecules desorbing from the solid surface, samples were carefully analyzed by considering the formation of carbonate species before and after analysis; therefore, data shown herein correspond to those adsorb on the surface and not coming from the carbonates formed. Table 3 shows the concentration of the basic sites (and also acid sites) of the Ca-Al mixed oxides. Because of the desorbed temperature, and according to the previous reports, ${ }^{26,27}$ these peaks could be assigned to oxygen anions, which desorb at temperatures higher than $773 \mathrm{~K}$. No other peaks were found in anywhere else temperature region (see Figure S2 in SI). Due to the absence of peaks $\mathrm{NH}_{3}$-TPD has not been included. 
Table 3. Concentration of basic and acid sites measured by $\mathrm{CO}_{2-}$ and $\mathrm{NH}_{3}$-TPD analysis of the $\mathrm{Ca}-\mathrm{Al}$ mixed oxides.

\begin{tabular}{ccc}
\hline Sample & $\mu \mathrm{mol} \mathrm{CO} / \mathrm{m}^{2}$ & $\mu \mathrm{mol} \mathrm{NH}_{3} / \mathrm{m}^{2}$ \\
\hline Ca-Al (0.8) & 4.3 & 0.6 \\
Ca-Al (2.4) & 142.0 & 0.0 \\
Ca-Al (3.0) & 181.0 & 4.0 \\
Ca-Al (3.4) & 185.0 & 0.0 \\
\hline
\end{tabular}

As it can be seen in Fig. S2 (in SI), strong basic sites dominate on the surface of all of the mixed oxides, which show a very high amount of basic sites, except (as might be expected) the Ca-Al (0.8) sample having the lowest amount of $\mathrm{Ca}$. A correlation was observed between $\mathrm{Ca} / \mathrm{Al}$ molar ratio and the amount of basic sites, as amount of $\mathrm{Ca}$ increases total basicity increases too. Nevertheless, it is important to bear in mind that no so high differences were found between $\mathrm{Ca}-\mathrm{Al}$ mixed oxides with $\mathrm{Ca} / \mathrm{Al}$ molar ratios $\geq 3.0$. It was also possible to correlate catalytic activity with these basicity values, which is going to be discussed in the next section of the manuscript.

The SEM micrographs of the as-produced HT (3.0) precursor and the Ca-Al (3.0) mixed oxide are shown in Figures $3 a$ and $3 b$, respectively. The rest of the HTs and mixed oxides micrographs are not showing because of their similarity (see Figure S3 in $\mathrm{SI}$ ). The micrograph in Fig. 3a shows the layers typical of a HT-type phase, while after calcined these layers are collapsed because the dehydration at high temperatures (Fig. 3b). ${ }^{27}$ In addition, hexagonal sheets are also detected (red cycle in Fig. 3a). The successful homogeneous distribution of $\mathrm{Ca}$ and $\mathrm{Al}$ in the solid was confirmed by SEM-EDX mapping (see Fig. $3 \mathrm{c}$ ).
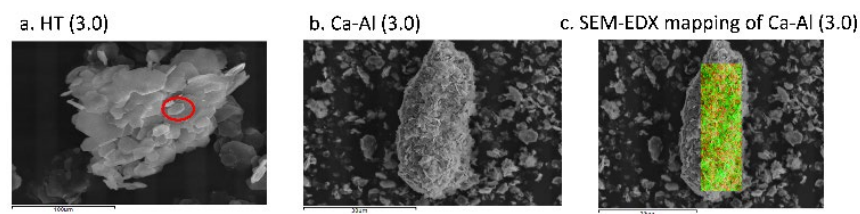

green $\mathrm{Ca}$, red $\mathrm{Al}$

Figure 3. SEM images of a. HT (3.0) before calcination; b. Ca-A (3.0) mixed oxide (after calcination); and c. SEM-EDX mapping of mixed oxide Ca-Al (3.0), Al (red) Ca (green).

\section{Glucose isomerization and epimerization over Ca-Al mixed oxides} A. Glucose-to-Fructose isomerization

In order to minimize residues and energy, water was used as solvent by working under mild reaction conditions (temperature $=373 \mathrm{~K}$ and pressure $=10$ bars of $\mathrm{N}_{2}$ ). Usually this reaction is performed at low glucose concentration, ${ }^{12}$ but going one step forward and in order to maximize product yield with a view to a future industrial application, a concentration higher or equal than $0.5 \mathrm{~mol} / \mathrm{L}$ was used. Table 4 shows the catalytic activity of Ca-Al mixed oxides here prepared at similar reaction conditions.
Table 4. Catalytic activity of Ca-Al mixed oxides in the isomerization of glucose. ${ }^{a}$

\begin{tabular}{ccccc}
\hline Catalysts & $\begin{array}{c}\text { Conversion } \\
\text { (\%) }\end{array}$ & $\begin{array}{c}\text { Fructose } \\
\text { Yield / } \\
\text { Selectivity (\%) }\end{array}$ & $\begin{array}{c}\text { Mannose } \\
\text { Yield / } \\
\text { Selectivity (\%) }\end{array}$ & $\begin{array}{c}\mathrm{C} \\
\text { Balance } \\
(\%)\end{array}$ \\
\hline $\mathrm{CaO}^{\mathrm{b}}$ & 59 & $26 / 49$ & $7 / 12$ & 57 \\
$\mathrm{Al}_{2} \mathrm{O}_{3}{ }^{\mathrm{b}}$ & $<1$ & $<1 / 98$ & $0 / 0$ & 97 \\
\hline $\mathrm{Ca}-\mathrm{Al}(0.8)$ & 35 & $32 / 94$ & $<1 / 8$ & 95 \\
$\mathrm{Ca}-\mathrm{Al}(2.4)$ & 41 & $30 / 74$ & $8 / 21$ & 92 \\
$\mathrm{Ca}-\mathrm{Al}(3.0)$ & 50 & $43 / 87$ & $6 / 13$ & 99 \\
$\mathrm{Ca}-\mathrm{Al}(3.4)$ & 89 & $23 / 26$ & $65 / 73$ & 98 \\
\hline
\end{tabular}

${ }^{a}$ Reaction conditions: [Glucose] $=0.55 \mathrm{M}, 0.05 \mathrm{~g}$ catalyst, $5 \mathrm{~mL} \mathrm{H} \mathrm{H}_{2} \mathrm{O}, \mathrm{PN}_{2}=10$ bar, temperature $=363 \mathrm{~K}$, reaction time $=90 \mathrm{~min} .{ }^{\mathrm{b}} \mathrm{Commercial}$ samples.

First, single oxides (i.e. $\mathrm{CaO}, \mathrm{Al}_{2} \mathrm{O}_{3}$ ) were employed to perform the isomerization of glucose, as shown in Table 4. The reaction with commercial $\mathrm{CaO}$ achieves good conversion but poor selectivity towards fructose, with a low carbon balance. Aluminum oxide did not give activity getting less than $1 \%$ of glucose conversion with good selectivity to fructose and carbon balance. Performing the isomerization of glucose with the Ca-Al catalysts containing different amounts of $\mathrm{Ca}$ leads to different results based on the Ca:Al molar ratio of the sample. As Ca:Al molar ratio increases conversion of glucose increases reaching almost a quantitative conversion [Ca-Al (3.4)]. However, selectivity towards fructose does not follow the same trend. Good selectivity to fructose was achieved by using the catalysts with Ca:Al molar ratio between 0.8 to 3.0. Nevertheless, increasing the Ca:Al molar ratio $>3$ promotes changes in the activity of the catalysts and the production of mannose was enhanced, thus reaching a mannose selectivity of $73 \%$. By contrast, the lowest Ca:Al molar ratio gives the highest selectivity to fructose, although the conversion of glucose (35\%) was the lowest [Ca-Al (0.8)].

As can be seen from the data of Table 4 and under the same reaction conditions (at $363 \mathrm{~K}$ and 10 bars of $\mathrm{N}_{2}$ ), the catalysts with Ca:Al molar ratio between 0.8 to 3.0 afforded quite similar yields of fructose $32-43 \%$, because all of them reach the equilibrium of glucose isomerization. Therefore, the initial isomerization rates, fare from the equilibrium, were investigated in order to make a proper comparison and choose the best catalyst for next studies. Figure 4 shows a correlation between isomerization rate, expressed as $\mathrm{mmol}$ fructose $\cdot \mathrm{g}^{-1} \cdot \mathrm{h}^{-1}$ in function of the Ca:Al molar ratio of catalysts. It is easy to note the increasing in reaction rate as $\mathrm{Ca}$ content in the solids increase. Due to the fact that the fastest catalyst [Ca-Al (3.4)] gives mannose as main product, we decided to choose the $\mathrm{Ca}$ Al (3.0) sample for next studies. In order to accurate the reliability of the results, the standard deviation for each group of experiments was calculated getting values from 0.007 to 0.0005 (see Figure S4 in SI). 


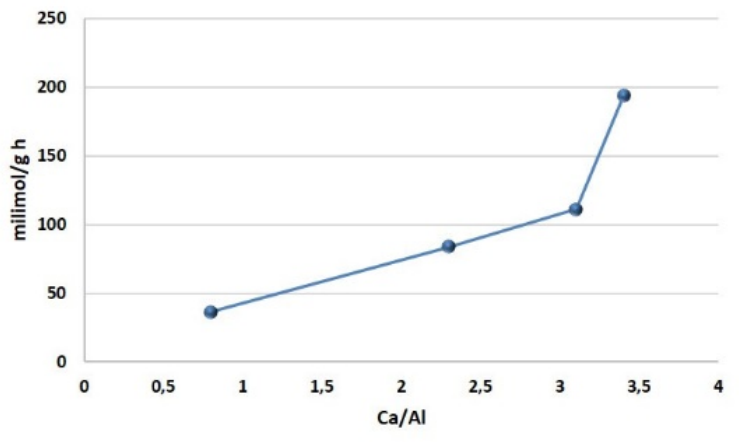

Figure

4. Correlation between Ca:Al molar ratio of catalysts and glucose isomerization rate.

Turnover frequencies, TOF $\left(\mathrm{h}^{-1}\right)$, for the different catalysts essayed in the glucose isomerization were calculated from the initial isomerization rate divided by the density of basic sites (see Table 3). An interesting correlation exists between fructose selectivity and TOF, as shown in Figure 5.

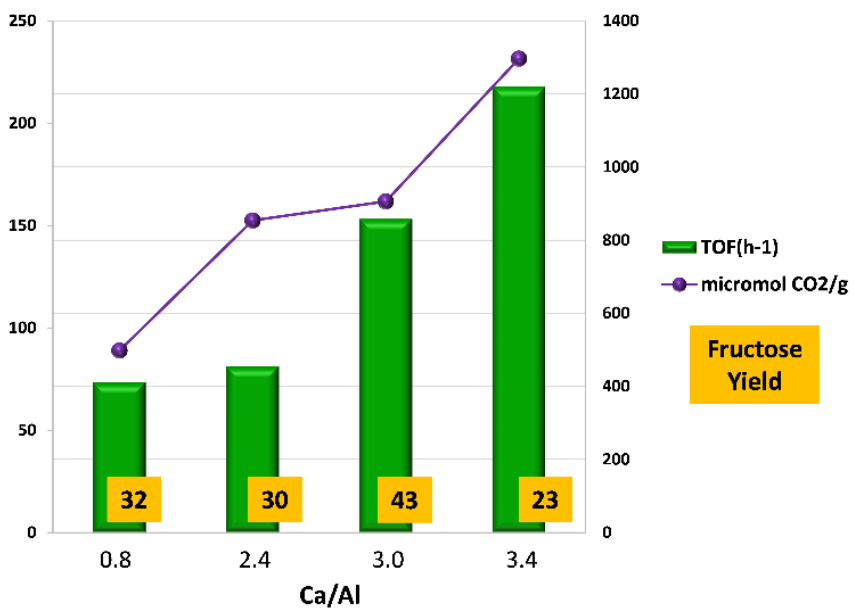

Figure 5. Correlation between TOF, fructose yield and basicity (in terms of $\mu \mathrm{mol} \mathrm{CO}_{2} / \mathrm{g}$ ) vs Ca content of the solids.

Some interesting conclusions could be extracted from the data given in Figure 5, such as:

a. Highest yield of fructose was observed using the Ca-Al (3.0) catalyst having a high density of basic sites (c.a. $906 \mu \mathrm{mol} / \mathrm{g}$ ), and a few amount of acids (c.a. $20 \mu \mathrm{mol} / \mathrm{g}$ ). Therefore, the adequate combination of basic and acid sites appears to be crucial to give a good performance on the isomerization of glucose to fructose.

b. A very high density of basic sites leads to the highest TOF but also to a low selectivity towards fructose, as can be seen in the case of Ca-Al (3.4) catalyst, owing to the formation of mannose as the main product of reaction. This highest mannose production was achieved with the catalyst possessing the highest density of basic sites and no acid functionalities. In addition, the presence of $\mathrm{CaCO}_{3}$ in the $\mathrm{Ca}-\mathrm{Al}$ (3.4), as it can be seen in its XRD pattern, can help in the formation of mannose.
To clarify this last conclusion, a reaction in presence of $\mathrm{CaCO}_{3}$ was performed, together with the catalyst that have no activity towards mannose, this means Ca-Al (0.8) sample. The production of mannose was enhanced achieving from $<1 \%$ to $10 \%$ of yield with $\mathrm{CaCO}_{3}$ addition. The same experiment was performed using the Ca-Al (3.0) catalyst, also detecting $\approx 20 \%$ increase of mannose yield with $\mathrm{Ca}$ salt addition. From these results it can be concluded that the presence of $\mathrm{CaCO}_{3}$ together with the high basicity and the lack of acidity observed for these solids play a key role in the epimerization of glucose to mannose.

In addition, it is important to note the kind of basic sites present in this solids, all of them have strong basicity characterized by the presence of peaks at high temperature $\geq 550^{\circ} \mathrm{C}$ detected by means of $\mathrm{CO}_{2}$-TPD analysis (see Figure $\mathrm{S} 2$ in $\mathrm{SI}$ ), which can be assigned to oxygen anions according to the literature. ${ }^{28}$ Strong basic sites dominate the surface of the solids, however the solids having the intermediate (3.0) and the highest (3.4) Ca:Al molar ratios, exhibit two different peaks (Fig. S2 in SI) assigned to the presence of strong and intermediate-strength basic sites in both solids. Peak for intermediate-strength basicity is more predominant in the case of Ca-Al (3.4) sample, also having the highest total basicity that leads to a very high yield of mannose, fact that could be explained by the need of a balance between strong/intermediate basicity to perform the epimerization, and a mixture of strong/intermediate sites, being the strong predominant, probably together with some few amounts of acid sites to perform the isomerization to fructose. ${ }^{29}$

Despite the large surface area of the Ca-Al (0.8), it did not show a superior activity in the isomerization of glucose, mainly in terms of conversion. The activity of the Ca-Al-based catalysts is mostly determined by the chemical composition and type of the material, which might offer the adequate and accessible basic sites to carry out the desired reaction.

Taking into consideration that Ca-Al (3.0) exhibited one of the best catalytic performance for glucose-to-fructose isomerization and a high isomerization rate $\left(2313 \mathrm{mmol} / \mathrm{g}_{\mathrm{cat}} \mathrm{h}\right)$, the influence of varying temperature and pressure of the process was then evaluated. Figure 6 shows the catalytic performance (in terms of glucose conversion and selectivity to fructose) on the different reaction conditions tested by using Ca-Al (3.0) catalyst.

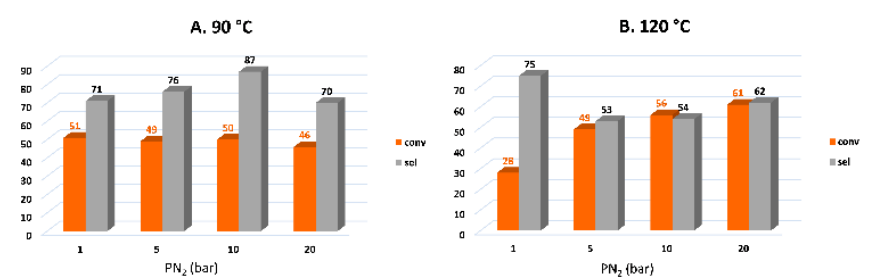

Figure 6. Reaction condition screening for the isomerization of fructose at A. $363 \mathrm{~K}$ and B. $393 \mathrm{~K}$.

On one hand, at $363 \mathrm{~K}$ glucose conversion kept constant when pressure was increased from 1 to 20 bar, while selectivity to 
fructose increased to reach a maximum of $87 \%$ at 10 bar of pressure; after that selectivity decreased when the pressure of reaction was augmented until 20 bar. On the other hand, an increase on the temperature (from 363 to $393 \mathrm{~K}$ ) let to a decrease in the selectivity to fructose, being some decomposition products detected in the reaction medium. Among all of the suitable decomposition products, glycolaldehyde (GLY) was detected in a higher proportion, by both HPLC (Figure S5 in SI) and ${ }^{13} \mathrm{C}-\mathrm{NMR}$ (Figure S6 in SI) measurements. These side reactions taking place during the isomerization of glucose are those typically observed in the presence of basic catalysts (see Scheme 2). ${ }^{30,33}$

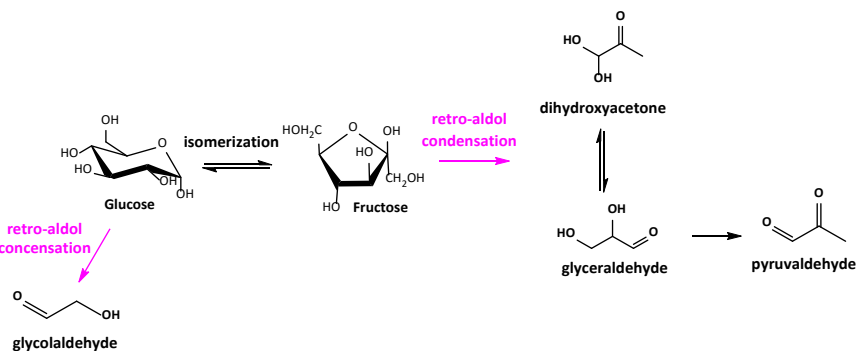

Scheme 2. Side reactions occurring during the isomerization of glucose to fructose.

Further investigations were developed in order to find out the role that glycolaldehyde GLY plays in the reaction. In this sense, a series of experiments in presence of GLY $(2 \mathrm{mmol})$ were done in the established optimal conditions (at $363 \mathrm{~K}$ and 10 bar of $\mathrm{N}_{2}$ ) using the Ca-Al (3.0) mixed oxide as catalyst. In these experiments, a decrease in both activity and selectivity towards fructose were observed (see Figure $\mathrm{S} 7$ in $\mathrm{SI}$ ); therefore, we can suggest that the presence of GLY in the reaction medium inhibits the formation of fructose, because its higher transformation ratio, as other authors have already postulated. ${ }^{11}$

Once the optimal reaction conditions were set (363 K and 10 bar of $\mathrm{N}_{2}$ ) and the most active catalyst, Ca-Al (3.0) was selected, reaction kinetics were further investigated to get some insights on the reaction mechanism (see Figure 7). Catalytic experiments were repeated several times to accurate the given values were correct (see Figure S18 in $\mathrm{SI}$ ). As can be seen, glucose conversion achieved around $50 \%$ at 90 min of reaction, and then it was kept constant over the time even if a small decrease in its value was observed mainly due to the deactivation of the catalyst, fact that will be discussed in more detail in the next section. Nevertheless, an unexpected profile, almost a sinusoidal graph, was obtained for the selectivity towards the two main products, fructose and mannose, respectively (HPLC of the points at 90 , 180 and $270 \mathrm{~min}$ are shown in Figure S8, in SI). From these results, it seems like glucose is firstly isomerized to fructose achieving a maximum selectivity of $88 \%$, point where the equilibrium is reached; therefore, the reaction does not evolve in this direction (towards the production of fructose), although the catalyst continues to be active. Thus, the question to ask now is if mannose is consecutively formed via the isomerization of fructose, the most logical pathway or rather, fructose is isomerized to glucose and then the epimerization takes place forward mannose (see Scheme 3).

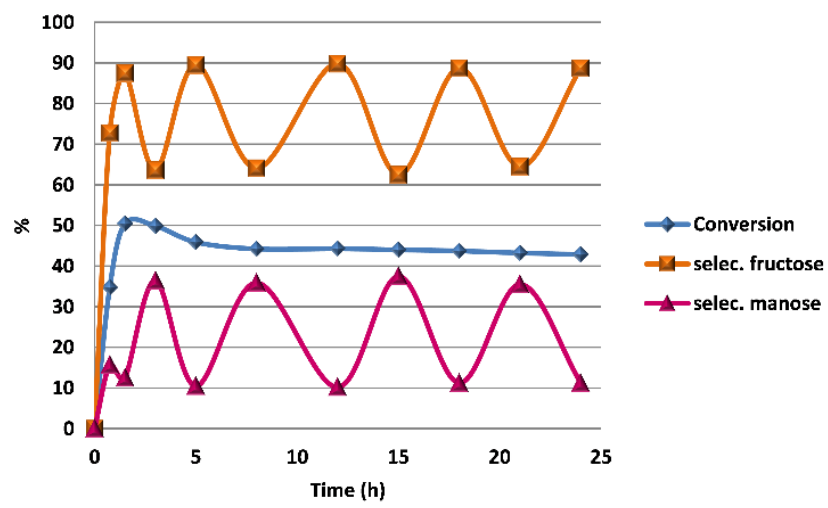

Figure 7. Kinetics of the isomerization of glucose-to-fructose catalyzed by Ca-Al (3.0).

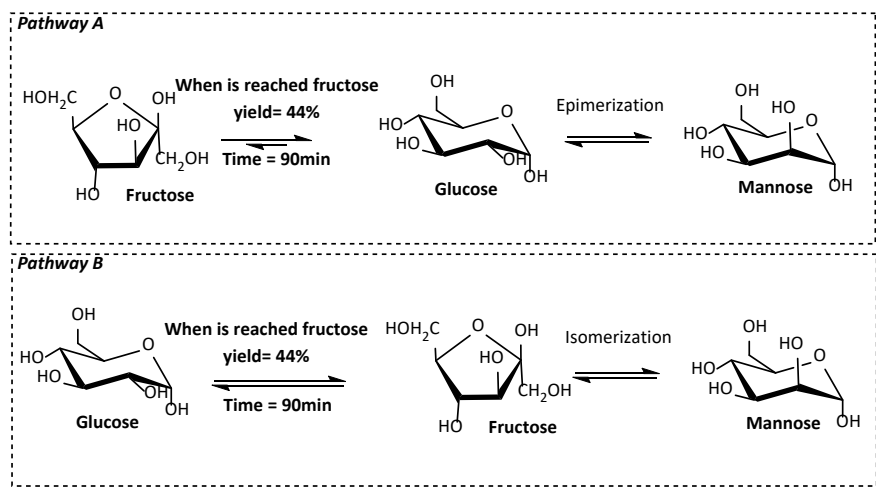

Scheme 2. Possible pathways in the production of mannose from glucose or fructose.

In order to elucidate this complicate issue some reactions having fructose as starting reactant were performed, and the results are shown in Table 5 .

Table 5. Catalytic activity of Ca-Al (3.0) with fructose as substrate.

\begin{tabular}{ccccccc}
\hline Entry & $\begin{array}{c}\text { Time } \\
(\mathbf{m i n})\end{array}$ & $\begin{array}{c}\text { Temperature } \\
\text { (K) }\end{array}$ & $\begin{array}{c}\text { Conversion } \\
\text { (\%) }\end{array}$ & $\begin{array}{c}\text { Glucose } \\
\text { Yield } \\
(\%)\end{array}$ & $\begin{array}{c}\text { Mannose } \\
\text { Yield (\%) }\end{array}$ & $\begin{array}{c}\text { GLY } \\
\text { Yield } \\
(\%)\end{array}$ \\
\hline 1 & 45 & & 41 & 34 & 7 & 7 \\
2 & 180 & \multirow{2}{*}{363} & 53 & 34 & 11 & 7 \\
3 & 210 & & 61 & 38 & 15 & 8 \\
4 & 300 & & 63 & 36 & 17 & 7 \\
\hline 5 & 45 & & 61 & 37 & 12 & 11 \\
6 & 180 & 393 & 87 & 38 & 39 & 10 \\
7 & 210 & & 87 & 35 & 40 & 11 \\
\hline
\end{tabular}

Two different temperatures were checked, 363 and $393 \mathrm{~K}$, respectively. At $363 \mathrm{~K}$ mainly glucose was detected as product (entries 1-4 in table 5), but it is interesting to highlight that 
conversion increased with the time of reaction, as well as the mannose yield, while a decrease in the glucose concentration was observed at the same time. When increasing the temperature $(393 \mathrm{~K})$, conversion and mannose production were increased, thus observing the same tendency as the reaction performed at low temperatures: an increase in the mannose yield is observed with the reaction time. To corroborate that mannose was not formed by the isomerization of fructose, liquid samples were taken off from the reaction mixture and analyzed at very short reaction times. Glucose and mannose yields obtained at very short reaction times are drawn in Figure 8 . At the beginning of the reaction, fructose is mainly isomerized to glucose, and then, when the glucose concentration in the reaction medium is enough, glucose epimerization to form mannose takes place. In light of all of these results it is reasonable to conclude that mannose is mostly formed by the epimerization of glucose more than by the isomerization of fructose, although this latest pathway cannot be discarded entirely.

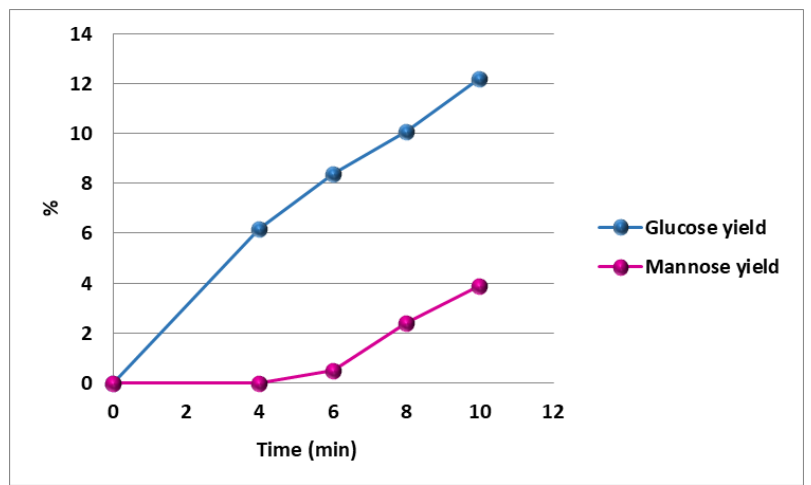

Figure 8. Glucose and mannose yields at short reaction times using fructose as substrate.

\section{B. Glucose-to-Mannose epimerization.}

Taking into account that the catalyst having the highest calcium content, Ca-Al (3.4) showed the highest selectivity towards mannose, the performance of this catalyst in the selective epimerization of glucose was investigated by carrying out the kinetics of the reaction (see Figure 9). Catalytic experiments were repeated several times to accurate the given values were correct (see Figure S15 in SI).

Similar trends as before observed with glucose-to-fructose isomerization can be stated, with the difference that when mannose reached the maximum production (at $90 \mathrm{~min}$ ), conversion is also reaching the maximum as well. After that, both conversion and selectivity to mannose fell, and simultaneously an increase in fructose selectivity is observed. Therefore, we have two parallel equilibriums taking place: one between glucose and mannose, and the other between glucose and fructose. At the beginning of the reaction (time $<50 \mathrm{~min}$ ), glucose is isomerized to fructose and also epimerized to mannose, the former reaction rate being higher than that of the glucose epimerization. When conversion of glucose reached around $45 \%$, and although initially mannose selectivity started to decrease slightly, the equilibrium between glucose and fructose is achieved (time $=\mathbf{4 5} \mathrm{min}$ ); therefore, the process is reversed to glucose production and then glucose-to-mannose epimerization occurs until reaching a maximum in both glucose conversion ( $\approx 90 \%)$ and mannose selectivity ( $\approx 70 \%)$. Afterwards, the equilibrium between glucose and mannose is achieved, so it is once again reversed to glucose and then the isomerization to fructose takes place. Taking into account all these results together with those shown in Table 5 and Figure 7, it could be stated that fructose is isomerized to glucose and then under the correct basic environment epimerization takes places forming mannose. In basic medium, glucose isomerization into fructose requires little reorganization of the intermediate, whereas formation of mannose takes place through rotation around the C2-C3 bond (Scheme 4). ${ }^{34}$ The rotation is connected with substantial reorganization of the water shell, and thus, mannose is formed more slowly than fructose. ${ }^{35,36}$ If the catalyst has only strong basic sites the evolution to mannose would be more slowly because of the stronger coordination to the metal center. On the contrary, if this solid has also medium strength basic, as in the case of Ca-Al (3.4) sample, this coordination becomes weak making easier the rotation around $\mathrm{C} 2-\mathrm{C} 3$.

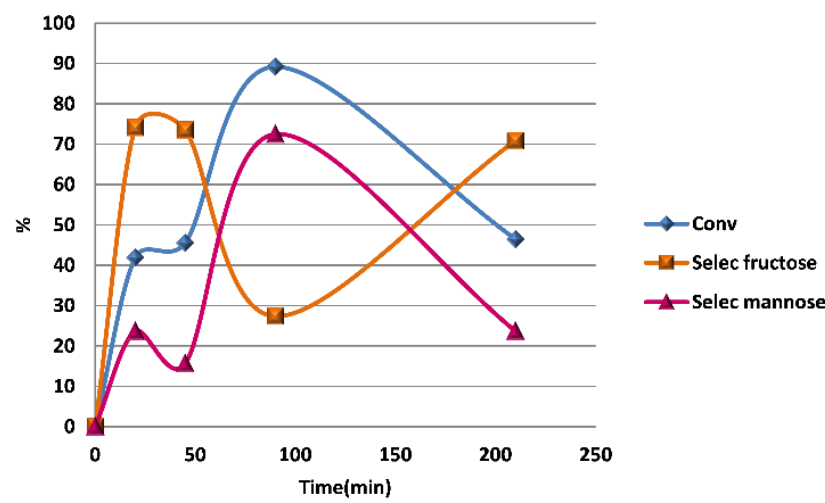

Figure 9. Kinetics of the epimerization of glucose using Ca-Al (3.4) as catalyst.

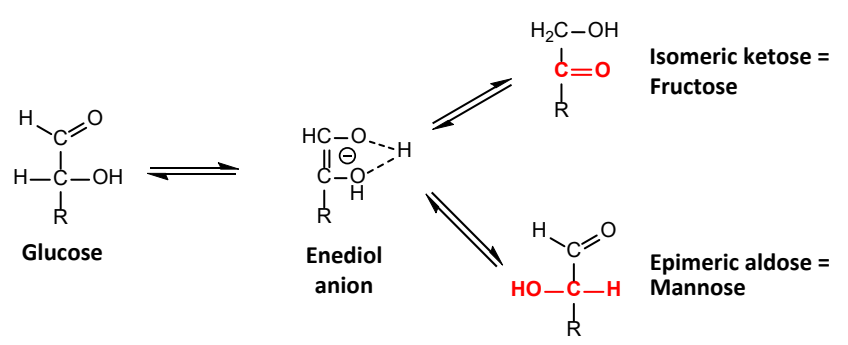

Scheme 4. Fructose and Mannose formation via enediol anion intermediate.

Taking into account the kinetics observed in Figures 7 and 9, rate constants for the glucose isomerization to fructose $(k 1)$ and for the glucose epimerization to mannose were calculated, obtaining the following values: $\mathrm{k} 1=0.020 \mathrm{~min}^{-1}$ and $\mathrm{k} 2=0.021$ $\mathrm{min}^{-1}$ (see Figures S16 and S17 in SI). As both constants have 
practically the same value, this could be consistent with the kinetic model of Competitive First Order Reactions, where the equations (developed by a well-known mathematical model) allow to calculating the fructose and mannose concentrations during the process (see $\mathrm{SI}$ ).

In order to get more insights in the mechanism of the process, the reaction using glucose-2-d1 as substrate was performed in the same conditions as those described for the isomerization of glucose to fructose with unlabeled glucose. In such a way, it was possible to elucidate the rate-determining step in the glucose isomerization due to the decrease in the isomerization rate when using glucose-2-d1. This indicates the $\mathrm{C} 2-\mathrm{H}$ bond cleavage as the rate-determining step of the process, as can be seen in Table S7 (in SI). Further NMR spectroscopic studies were performed to clarify the mechanism, but the results were not conclusive (see SI). Apparently, both mechanisms, basic and acid-catalyzed glucose isomerization could be involved, the first mechanism being the more plausible for our catalytic system.

\section{Catalysts recyclability}

One of the great advantages of the glucose isomerization process when carried out heterogeneously consists in its simple catalyst separation and reutilization, an essential requisite to make any process economically viable. The recyclability of Ca-Al (3.0) catalyst in the glucose isomerization to fructose and of $\mathrm{Ca}$ Al (3.4) catalyst in the glucose epimerization to mannose was evaluated by reusing the solids in several consecutive runs (see Figure 10). In a typical experiment and at the end of reaction, the solid catalyst was separated from the solution after previous centrifugation of the reaction mixture and ulterior filtration, then washed three times with water, and finally reused in a new catalytic experiment. A decrease in the activity of the catalysts was observed during the first re-use in both cases. After checking the recovered solids by XRD measurements, it was detected that hydrotalcite-type structure was regenerated (see Figure S9, in SI), this structure showing less activity towards isomerization than the $\mathrm{Ca}-\mathrm{Al}$ mixed oxides. With the aim to raise the catalytic activity again, a thermal treatment (calcination) of the recovered catalysts before the new run was made. In this way, the activity was almost recovered in the second re-use and maintained constant up to five consecutive re-uses, although a small decay is observed compared to the initial fresh catalyst. It is worth to note that when the catalyst loses its activity (mainly when it was re-used without previous calcination treatment) glycolaldehyde (GLY) was generated as main side-product.

By considering the results of Figure 10 and in order to accurate that hydrotalcite-type structure is less active towards both isomerization and epimerization of glucose, some additional experiments using hydrotalcite-type materials as catalysts were performed. Indeed, a decrease in the glucose conversion and the selectivity to the desired products was observed by using HT (3.0) and HT (3.4) for glucose isomerization and epimerization, respectively. Thus, glucose conversion of $15 \%$ and selectivity to fructose of $62 \%$ were achieved for isomerization to fructose, while glucose conversion of $45 \%$ and selectivity to mannose of $61 \%$ were reached for epimerization to mannose, being both results lower than the values achieved with the catalysts used in a new run or catalytic re-use without calcination treatment of the solids.

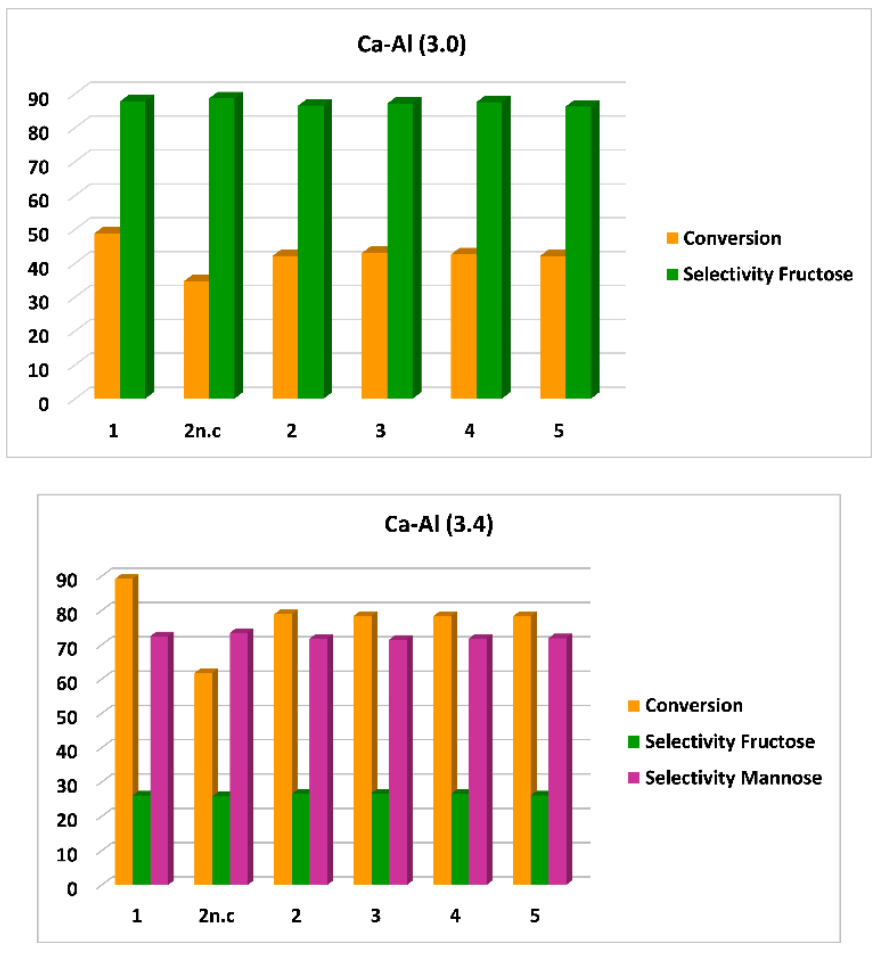

Figure 10. Repeated uses of Ca-Al-based catalysts for both A. Glucose isomerization to fructose and B. Glucose epimerization to mannose before and after regeneration (2n.c means noncalcined material). Reaction conditions: [Glucose $]_{i}=0.55 \mathrm{M}, 0.05$ g catalyst, $5 \mathrm{~mL} \mathrm{H} \mathrm{H}_{2} \mathrm{O} \mathrm{PN}_{2}=10$ bar, at $363 \mathrm{~K}$.

During the catalytic re-cycling tests, a small leaching of Ca was detected by checking the solids after the first and the last (fifth) run by means of ICP analysis (see Table S1 in SI). The measured loss of $\mathrm{Ca}$ (with respect to the total initial amount of $\mathrm{Ca}$ in the fresh solids) for both Ca-Al (3.0) and Ca-Al (3.4) catalysts was of $2.5 \%$ and $2.8 \%$, respectively, values given after the first and fifth runs being practically identical, this meaning that the principal leaching of Ca species occurs in the first run with a practically negligible increase in the value after several re-uses. In order to elucidate a possible catalytic activity of the leached $\mathrm{Ca}^{2+}$ ions, an additional test with hot filtration step was carried out. Thus, the catalytic reaction was conducted during $45 \mathrm{~min}$ in the presence of Ca-Al (3.0) sample until the conversion of glucose reached ca. $35 \%$. After that, the solid catalyst was removed by filtration and the filtrate was held under typical reaction conditions during another $45 \mathrm{~min}$. No changes in the concentrations of glucose or fructose could be detected by HPLC analysis, these evidences supporting that the isomerization of glucose is catalyzed heterogeneously over the Ca-Al-based catalysts. However, after two hours of incubation of the reaction mixture in the absence of the solid catalyst, the $\mathrm{pH}$ of the filtrate decreased to a more acid value confirming that $\mathrm{Ca}^{2+}$ species are not active for the isomerization reaction, but can catalyze the decomposition of carbohydrates into acidic by-products getting a high concentration of saccharic acid, as it was checked by HPLC analysis of the liquids at the end of the reaction. Similar results were obtained by other authors when a solution of $\mathrm{NaOH}$ was used as homogeneous catalyst. ${ }^{37}$ 
It is clear that our reaction conditions are so far from the physiological ones employed with enzymatic systems where proportions of glucose/fructose/mannose below the equilibrium values are reported. ${ }^{12}$ In addition, the conversion of glucose during epimerization attained with our catalytic system is so high because glucose is transformed into two products in parallel reactions, being the yield of fructose far from the equilibrium. Other authors observed something similar in their systems, ${ }^{38}$ glucose conversion increased in the reaction course because of the consumption of fructose; although this fructose transformation was not clear in the paper, since any other byproduct was detected in the reaction mixture.

More interestingly, it was also reported that using $\mathrm{Ca}(\mathrm{OH})_{2}$ as catalyst for this reaction, mannose yields superior to the thermodynamic equilibrium values can be reached. ${ }^{39}$ Authors explain that the formation of a complex between $\mathrm{Ca}^{2+}$ species and mannose allows getting a kinetic (instead of thermodynamic) control of the system, thus surpassing the $50 \%$ mannose yield theoretically expected. Taking into account that the Ca-Al (3.4) catalyst possesses a high amount of $\mathrm{Ca}^{2+}$ species exposed on the solid surface, the possibility of this complex formation at the solid-liquid interface could not be ruled out This does not mean that the $\mathrm{Ca}^{2+}$ species are leached from solid to the solution during reaction. In fact, as it was clearly demonstrated above, most of the $\mathrm{Ca}$ remains in the solid catalyst even after fifth consecutive catalytic cycles.

\section{Catalytic performance of Ca-Al-based materials in the isomerization of other sugars}

With the aim to explore the performance of these Ca-Al-based catalysts more in deep, the isomerization of other biomassderived sugars was performed. Xylose and lactose were chosen for this studies since: i) its isomerization still represents a challenging task; $;^{40-42}$ and ii) because their respective isomers are useful platform molecules for the synthesis of high addedvalue products, thus increasing at the same time the catalysts applicability. ${ }^{43}$

Table 6. Isomerization of xylose and lactose over Ca-Al-based catalysts. ${ }^{a}$

\begin{tabular}{ccccccc}
\hline Entry & Substrate & Catalyst & $\begin{array}{c}\text { Time } \\
(\mathbf{m i n})\end{array}$ & $\begin{array}{c}\text { Conversion } \\
(\%)\end{array}$ & $\begin{array}{c}\text { Isomer } \\
\text { select. } \\
(\%)\end{array}$ & $\begin{array}{c}\text { C } \\
\text { balance } \\
(\%)\end{array}$ \\
\hline 1 & & Ca-Al & 90 & 65 & 70 & 85 \\
2 & & $(0.8)$ & & & 21 & 81 \\
3 & xylose & Ca-Al & 90 & 45 & 18 & 74 \\
4 & & $(3.0)$ & 180 & 63 & 12 & 84 \\
5 & & Ca-Al & 90 & 42 & 9 & 72 \\
\hline 6 & & $(3.4)$ & 180 & 55 & 21 & 48 \\
7 & & Ca-Al & 90 & 45 & 72 & 98 \\
8 & lactose & Cal-Al & 90 & 65 & 72 \\
9 & & $(3.0)$ & 180 & 75 & 55 & 82 \\
10 & & Ca-Al & 90 & 53 & 95 & 98 \\
\hline
\end{tabular}

a Reaction conditions: [Substrate] $i=0.5 \mathrm{M}, 10 \mathrm{wt} \%$ of catalyst, $5 \mathrm{~mL} \mathrm{H} \mathrm{H}_{2}$, $\mathrm{PN}_{2}=10$ bar, at $363 \mathrm{~K}$.
The Ca-Al-based catalysts prepared in this study were tested in the isomerization of xylose-to-xylulose and lactose-to-lactulose, and the attained results are shown in Table 6. On one hand, the Ca-Al (3.0) catalyst was able to isomerize both substrates (xylose and lactose), but with lower catalytic performance compared to that reached in the glucose-to-fructose isomerization. Particularly, for the isomerization of xylose, results were as expected since the isomerization of xylose to xylulose had been classically performed by acid catalysts. ${ }^{44}$ In fact, when the isomerization reaction was performed in presence of a more acid Ca-Al mixed oxide, such as Ca-Al (0.8) (Entry 1), better values of conversion and selectivity were achieved. More interestingly, longer reaction times let to a decrease in the selectivity of the isomer and non-identified degradation products were obtained. On the other hand, different and better results were obtained in the case of the isomerization of lactose. Good selectivity to lactulose and lactose conversion were reached at $90 \mathrm{~min}$ of reaction time by using the Ca-Al (3.0) catalyst (Entry 7). By increasing the Ca:Al molar ratio from 3.0 to 3.4 , the catalytic activity increase too, achieving good conversion (53-56\%) and excellent selectivity to the isomer (95-96\%) by working at different reaction times (Entries 9, 10), although no more improvements were observed at longer reaction times. Remarkably, these results are better than those found in the bibliography, from our knowledge. These results represent a step forward in the isomerization of lactose since this reaction is usually performed with the addition of external bases or by using homogeneous catalysts, thus making difficult their recovering and recyclying. ${ }^{40}$ In addition, any cleavage or decomposition products were detected in the reaction mixture, while the corresponding epimer was also not detected in any reaction. Due to these very promising results, further investigations on the selective isomerization of lactose over Ca-Al-based materials are currently on-going in our research group.

\section{Green metrics: comparison with other systems}

Green metrics is a useful tool to evaluate the sustainability of a process and to make a fair comparison with the classical or already developed systems. Among all of the tools used for this purpose, we have chosen three:

1. Atom economy $(\mathrm{AE}):^{45,46} \mathrm{It}$ is the classical tool and is defined as the ratio of the molecular weight of the target molecule to the sum total of the molecular weights of all the substances produced in the stoichiometric equation for the reaction involved. It takes into account the amount of the reagents incorporated into the end product (see SI).

2. Environmental factor (E-factor) $:^{47} \mathrm{It}$ is the ratio of the weight of generated waste to the total weight of the end product. It is a useful tool for rapid evaluation of processes based on generated waste (see SI).

3. Ecoscale: 48 It is a simplified algorithm for evaluation of chemical reactions on laboratory scale developed by Koen Van Aken et al. It is a semi-quantitative tool that unifies economic and ecological aspects of a given reaction (see $\mathrm{SI}$ ).

Analysis of green metrics have been done for our catalytic system using Ca-Al-based materials by making a comparison with the enzymatic system used at industrial level (with xylose 
isomerase as catalyst), ${ }^{49}$ and one of the most cited heterogeneous system that uses $\mathrm{Sn}$-Beta zeolite as catalyst. ${ }^{50}$ Figure 11 shows a comparative estimation of the three green parameters for the three selected isomerization systems (see SI).

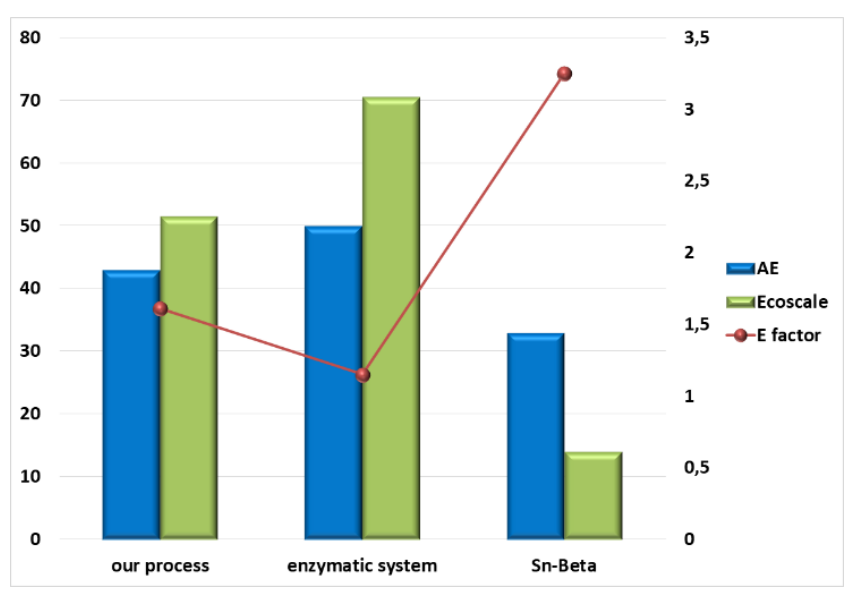

Figure 11. Comparison of $A E, E$-factor and Ecoscale, green parameters for our system and other two systems used in the glucose-to-fructose isomerization.

Comparison made in Figure 11 clearly shows that it is possible to compare our catalytic system with the enzymatic system in terms of AE parameter, even taking into account the limitations of the process itself where $50 \%$ conversion would be the maximum value. With respect to $\mathrm{E}$-factor parameter our system has not a bad number (considering the ideal E-factor of zero), value being higher than the enzymatic system, but much better than Sn-Beta system. In terms of a more general scale, the Ecoscale shows that the enzymatic system is better than our system (with a difference of 18.3 points), but it should be taken into account that for this calculation the need of using inert atmosphere in the synthesis of the catalysts was considered in our system. However, our catalytic process could be considered as a green process and at least greener than the process catalyzed by $\mathrm{Sn}$-Beta. In conclusion, our process is within the green concept, better than other systems based in heterogeneous catalysts, and close to the industrially used, xylose isomerase enzymatic route.

\section{Experimental}

\section{Chemicals:}

Glucose and fructose (Sigma-Aldrich, 99.5\%), lactose and xylose (Sigma-Aldrich, 99.9\%) were used as reagents in the isomerization and epimerization tests. For the analysis, mannose (99.99\%), galactose (99.99\%), glycolaldehyde dimer (99.99\%), glyceraldehyde ( $\geq 90 \%)$, xylulose $(\geq 98 \%)$, and lactulose $(\geq 95 \%)$, were purchased from Sigma-Aldrich and used as received. Water (Milli-Q quality, Millipore) was used as solvent. Hydrotalcite-precursors were prepared by using the proper metallic precursor bought from Sigma-Aldrich: $\mathrm{Ca}\left(\mathrm{NO}_{3}\right)_{2} \cdot 4 \mathrm{H}_{2} \mathrm{O}$ ( $\geq 99 \%), \mathrm{Al}\left(\mathrm{NO}_{3}\right)_{3} \cdot 9 \mathrm{H}_{2} \mathrm{O}(\geq 98 \%)$, and an aqueous solution of $\mathrm{NaOH}(98 \%)$ in milli-Q water. Single oxides $\mathrm{CaO}$ and $\mathrm{Al}_{2} \mathrm{O}_{3}$ were purchased also from Sigma-Aldrich. Nitrogen (99.999\%) was acquired from Abelló Linde S.A.

\section{Catalysts preparation:}

Due to the few examples of this Ca-Al hydrotalcite-type materials found in bibliography, a complete optimization of their synthesis was performed. Optimization as well as XRD analysis of each sample is depicted in the Supporting Information (Table S6, and Figures S10-13). The hydrotalciteprecursors were prepared by adaptation of the co-precipitation method described in ref. ${ }^{19}$ Once main parameters of synthesis (i.e. aging temperature, base concentration, etc.) were optimized, the standard procedure for hydrotalcite-precursors preparation was as follows: Starting from two different solutions, solution $\mathrm{A}$, containing the metallic species $(\mathrm{Ca}, \mathrm{Al})$ in the desired concentrations and solution $\mathrm{B}$ containing the basic solution in the wanted concentration that is: $\left[\mathrm{OH}^{-}\right]=2\left[\mathrm{Ca}^{2+}\right]$. These two solutions were added at room temperature, at the same rate $(20 \mathrm{~mL} / \mathrm{h})$ to an empty flask under $\mathrm{N}_{2}$ atmosphere with a stirring speed of $500 \mathrm{rpm}$. The precipitates were aged for $18 \mathrm{~h}$ at $333 \mathrm{~K}$ in an oven, keeping the $\mathrm{N}_{2}$ atmosphere. After that, the solids were washed with deionized water until $\mathrm{pH} \leq 8$ and calcined, prior to their use in the catalytic experiments. The calcination treatment was done from room temperature until $723 \mathrm{~K}$ with a temperature rate of $2{ }^{\circ} \mathrm{C} / \mathrm{min}$ under air atmosphere, and then kept at $723 \mathrm{~K}$ during $4 \mathrm{~h}$ under $\mathrm{N}_{2}$ atmosphere (see Fig. S18 and comments in SI). Finally, the solids were cooling down during $8 \mathrm{~h}$ under $\mathrm{N}_{2}$ atmosphere.

\section{Catalysts characterization:}

HT-precursors and Ca-Al mixed oxides were characterized by inductively coupled plasma (ICP) with a Varian 715-ES ICPOptical emission spectrometer, after solid dissolved in $\mathrm{HNO}_{3}$ aqueous solution. Phase purity was determined by $\mathrm{X}$-ray diffraction (XRD) in a Philips X pert MPD diffractometer equipped with a PW3050 goniometer (CuKa radiation, graphite monochromator). Surface areas of the solid samples (150 mg) were calculated by applying the BET method to the $\mathrm{N}_{2}$ adsorption isotherms obtained by carrying out liquid nitrogen adsorption experiments at $77 \mathrm{~K}$, in a Micromeritics flowsorb apparatus.

The basicity of the catalysts was studied by temperatureprogrammed desorption of $\mathrm{CO}_{2} \quad\left(\mathrm{CO}_{2}-\mathrm{TPD}\right)$. Approximately $100 \mathrm{mg}$ of sample was pretreated with a stream of helium at $1073 \mathrm{~K}$ during $30 \mathrm{~min}(10 \mathrm{~K} / \mathrm{min}$ and $60 \mathrm{~mL} / \mathrm{min})$. The reaction temperature was then decreased to $373 \mathrm{~K}$, and a flow of pure $\mathrm{CO}_{2}(60 \mathrm{~mL} / \mathrm{min})$ was subsequently introduced into the reactor during $30 \mathrm{~min}$. The $\mathrm{CO}_{2}-\mathrm{TPD}$ reaction was carried out between 373 and $1073 \mathrm{~K}$ under a flow of helium $(10 \mathrm{~K} / \mathrm{min}, 30 \mathrm{~mL} / \mathrm{min})$, and the amount of $\mathrm{CO}_{2}$ evolved was analyzed using a quadrupole mass spectrometer (Balzer GSB 300 02) equipped 
with a Faraday detector (0-200 U), which monitors the mass of $\mathrm{CO}_{2}(44 \mathrm{U})$ during the experiment.

The temperature-programmed desorption of ammonia $\left(\mathrm{NH}_{3}-\right.$ TPD) was carried out to evaluate the total surface acidity of the catalysts. After cleaning of the catalysts $(80 \mathrm{mg})$ with helium and subsequent adsorption of ammonia at $373 \mathrm{~K}$, the $\mathrm{NH}_{3}$-TPD measurement was performed by raising the temperature from 373 to $823 \mathrm{~K}$, under a helium flow of $40 \mathrm{~mL} / \mathrm{min}$, with a heating rate of $10 \mathrm{~K} / \mathrm{min}$ and maintained at $823 \mathrm{~K}$ during $15 \mathrm{~min}$. The evolved ammonia was analyzed using a TCD detector of a gas chromatograph (ShimadzuGC-14A).

A scanning electron microscope (SEM, Zeiss instrument, AURIGA Compact) has been employed to determine the morphology of the solid samples before and after calcination. FT-IR spectroscopic analyses of the fresh catalyst samples were performed on a Perkin Elmer FT-IR spectrometer, and the spectra were recorded in the region of $4000-500 \mathrm{~cm}^{-1}$.

\section{Catalytic experiments:}

Catalytic tests were performed in a $12 \mathrm{~mL}$ stainless steel autoclave type reactor with PEEK (polyether-ethyl-ketone) interior, equipped with a magnetic bar, pressure control and a valve for either liquid or gas sample extraction, and an electrical heating jacket. Typically, $2.7 \mathrm{mmol}$ of the desired substrate (glucose: $0.5 \mathrm{~g}$; fructose: $0.5 \mathrm{~g}$ ) was dissolved in $5 \mathrm{~mL}$ of distilled water and placed in the reactor together with $0.05 \mathrm{~g}$ of the catalyst. The autoclave was then closed and purged three times with $\mathrm{N}_{2}$. Then, the reactor was charged with the appropriate pressure of nitrogen and heated to the desired temperature (363 or $393 \mathrm{~K}$ ). Stirring was stopped at fixed intervals of time and liquid samples $(0.1 \mathrm{~mL})$ were withdrawn and then analyzed by HPLC by following reactants and products evolution.

Recycling tests were performed using the desired catalysts at the fixed optimal conditions. After a catalytic run, the reaction mixture was centrifuged and the solid catalyst was recovered by filtration, then washed with ultrapure water and reused in a new run under the same conditions. Because a drop in the activity was observed, a previous calcination treatment was made by applying the same conditions as in the synthesis of the mixed oxides.

\section{Reaction mixtures analyses:}

The liquid samples collected from the experiments were analyzed using HPLC (Varian) equipped with a refractive index detector (RI) Varian Star with internal temperature of $323 \mathrm{~K}$ to determine the amount of products and substrates. Bio-Rad Aminex HPX-87H column was used in the case of glucose-tofructose (or mannose), while HPX-87P $(9 \mu \mathrm{m}, 7.8 \times 300 \mathrm{~mm}$ ) column was employed for xylose-to-xylulose and lactose-tolactulose reactions. In both cases, the columns were maintained at $333 \mathrm{~K}$ and the mobile phase was $100 \%$ milli-Q HPLC grade water at a flow rate of $0.5 \mathrm{~mL} / \mathrm{min}$. Analysis were done by using external calibration curves generated for all of the suitable products separately using standard solutions with known concentrations. The results are reported in terms of substrate conversion, and products yield and selectivity, which are calculated as follows:

Substrate conversion (\%) $=$ (Moles of substrate converted)/(Initial moles of substrate) $\times 100$

Products yield $(\%)=($ Moles of product produced $) /($ Initial moles of substrate) $\times 100$

Products selectivity $(\%)=($ Moles of product produced $) /($ Moles of substrate converted ) $\times 100$

\section{Conclusions}

In this work, some hydrotalcite-precursors and their derived mixed oxides containing $\mathrm{Ca}$ and $\mathrm{Al}$ were successfully prepared by a sustainable method and evaluated in the isomerization of various sugars. Hydrotalcite-type phase was obtained as the main crystalline phase in all the HT-precursors prepared with Ca:Al molar ratios ranging from 0.8 to 3.4 , and no rest of $\mathrm{CaCO}_{3}$ was observed in almost all of the solids, with the exception of the solid having the highest Ca:Al molar ratio [Ca-Al (3.4)], in which a $7.3 \%$ of $\mathrm{CaCO}_{3}$ was detected. The presence of this $\mathrm{CaCO}_{3}$ played a key role to tune the catalyst activity and drive the reaction to the production of mannose instead of fructose. A superior activity was observed in the glucose isomerization to fructose for the catalysts with an intermediate $\mathrm{Ca}$ :Al molar ratio and intermediate basicity [i.e. Ca-Al (3.0)]. More interestingly, these $\mathrm{Ca}-\mathrm{Al}$-based materials are capable to isomerize other sugars, such as lactose and xylose, thus extending their application scope. Recyclability of the catalysts was also evaluated for both isomerization and epimerization of glucose showing a good resistance under reaction conditions over several catalytic runs. Some green metrics were also evaluated, the here proposed catalytic system fits very well with green concepts compared with the enzymatic system, industrially employed, while showing superior sustainability than other systems based on solid acid catalysts.

\section{Conflicts of interest}

There are no conflicts to declare.

\section{Acknowledgements}

Financial support by the Spanish Government (PGC2018097277-B-I00 and SEV-2016-0683), Generalitat Valenciana (GVA, PROMETEO/2018/006), and especially the Unesco/IUPAC/PhosAgro partnership within the program "Green Chemistry for Life" (Grant \#139) is gratefully acknowledged. Authors also thank the ITQ for the technical support, and the Electron Microscopy Service of Universitat Politècnica de València for instrumental facilities. 


\section{Notes and references}

1 Y. Z. Mao, S. Y. Tian, Y. M. Qin and J. Z. Han, Food Chem., 2019, 281, 78-84.

2 M. Ventura, F. Nocito, E. De Giglio, S. Cometa, A. Altomare and A. Dibenedetto, Green Chem., 2018, 20, 3921-3926.

3 REN21, Journal, 2018, 210.

4 J. P. Casey, Starch - Stärke, 1977, 29, 196-204.

5 S. H. Bhosale, M. B. Rao and V. V. Deshpande, Microbiol. Mol. Biol. Rev., 1996, 60, 280-300.

6 I. K. M. Yu, X. N. Xiong, D. C. W. Tsang, L. Wang, A. J. Hunt, H. Song, J. Shang, Y. S. Ok and C. S. Poon, Green Chem., 2019, 21, 1267-1281.

7 H. J. Cho, N. S. Gould, V. Vattipalli, S. Sabnis, W. Chaikittisilp, T. Okubo, B. J. Xu and W. Fan, Micropor. Mesopor. Mat., 2019, 278, 387396.

8 I. Graça, M. C. Bacariza, A. Fernandes and D. Chadwick, Appl. Catal. B, 2018, 224, 660-670.

9 Z. Wang, in Comprehensive Organic Name Reactions and Reagents, 2010, DOI: 10.1002/9780470638859.conrr396.

10 Y. J. Pagán-Torres, T. Wang, J. M. R. Gallo, B. H. Shanks and J. A. Dumesic, ACS Catal., 2012, 2, 930-934.

11 A. A. Marianou, C. M. Michailof, A. Pineda, E. F. Iliopoulou, K. S. Triantafyllidis and A. A. Lappas, ChemCatChem, 2016, 8, 1100-1110. 12 I. Delidovich and R. Palkovits, ChemSusChem, 2016, 9, 547-561. 13 B. Kamm, Angew. Chem., 2007, 46, 5056-5058.

14 M. Ventura, D. Williamson, F. Lobefaro, M. D. Jones, D. Mattia, F. Nocito, M. Aresta and A. Dibenedetto, ChemSusChem, 2018, 11, 1073-1081.

15 M. Yabushita, N. Shibayama, K. Nakajima and A. Fukuoka, ACS Catal., 2019, 9, 2101-2109.

16 R. Bermejo-Deval, R. Gounder and M. E. Davis, ACS Catal., 2012, 2, 2705-2713.

17 M. L. Hayes, N. J. Pennings, A. S. Serianni and R. Barker, J. Am. Chem. Soc., 1982, 104, 6764-6769.

18 T. Tanase, F. Shimizu, S. Yano and S. Yoshikawa, ChemComm, 1986, DOI: 10.1039/C39860001001, 1001-1003.

19 C.-H. Wu, Y.-P. Chang, S.-Y. Chen, D.-M. Liu, C.-T. Yu and B.-L. Pen, J. Nanosci. Nanotechnol., 2010, 10, 4716-4720.

20 L. Gao, G. Teng, G. Xiao and R. Wei, Biomass Bioenerg., 2010, 34, 1283-1288.

21 Y. Li, J. Wang, Z. Li, Q. Liu, J. Liu, L. Liu, X. Zhang and J. Yu, Chem. Eng. J., 2013, 218, 295-302.

22 S. Witjes and R. Lozano, Resour. Conserv. Recyc., 2016, 112, $37-$ 44.

23 F. Cavani, F. Trifirò and A. Vaccari, Catal. Today, 1991, 11, 173301.

24 J. Plank, H. Keller, P. R. Andres and Z. Dai, Inorganica Chim. Acta, 2006, 359, 4901-4908.

25 G. A. Lager, R. T. Downs, M. Origlieri and R. Garoutte, Am. Mineral, 2002, 87, 642-647.

26 D. P. Debecker, E. M. Gaigneaux and G. Busca, Chem. Eur. J., 2009, 15, 3920-3935.

27 J. I. Di Cosimo, V. K. Díez, M. Xu, E. Iglesia and C. R. Apesteguía, J. Catal., 1998, 178, 499-510.

28 Lima, A. S. Dias, Z. Lin, P. Brandão, P. Ferreira, M. Pillinger, J. Rocha, V. Calvino-Casilda and A. A. Valente, Appl. Catal. A:Gen., 2008, 339, 21-27.

29 F. M. Labajos, V. Rives, P. Malet, M. A. Centeno and M. A. Ulibarri, Inorg. Chem., 1996, 35, 1154-1160.

30 K. Shekoohi, F. S. Hosseini, A. H. Haghighi and A. Sahrayian, MethodsX, 2017, 4, 86-94.
31 J. Zhang, B. Hou, A. Wang, Z. Li, H. Wang and T. Zhang, AlChE J., 2014, 60, 3804-3813.

32 Z. Tan, G. Miao, C. Liu, H. Luo, L. Bao, L. Kong and Y. Sun, RSC Adv. 2016, 6, 62747-62753.

33 G. De Wit, A. P. G. Kieboom and H. van Bekkum, Carbohydr. Res., 1979, 74, 157-175.

34 B. M. Kabyemela, T. Adschiri, R. M. Malaluan and K. Arai, Ind. Eng. Chem. Res., 1997, 36, 1552-1558.

35 M. Rellán-Piñeiro, M. Garcia-Ratés and N. López, Green Chem. 2017, 19, 5932-5939.

36 C. G. Yoo, N. Li, M. Swannell and X. Pan, Green Chem., 2017, 19, 4402-4411.

37 M. Wang, M. A. A. Gasmalla, H. Admassu Tessema, X. Hua and R. Yang, Food Chem., 2017, 233, 151-158.

38 R. Otomo, M. Fujimoto, M. Nagao and Y. Kamiya, Mol. Catal., 2019, 475, 110479-110487.

39 R. Yanagihara, K. Soeda, S. Shiina, S. Osanai and S. Yoshikawa. Bull. Chem. Soc. Jpn., 1993, 66, 2268-2272

40 M. Aider and D. d. Halleux, Trends Food Sci. Technol., 2007, 18 356-364

41 C. Moreno-Marrodan, P. Barbaro, S. Caporali and F. Bossola, ChemSuschem, 2018, 11, 3649-3660

42 V. Choudhary, S. Caratzoulas, D. G. Vlachos, Carbohydr. Res., 2013, 368, 89-95.

43 N. Seki and H. Saito, Int. Dairy J., 2012, 22, 110-115.

44 Q. Lin, Y. Yan, X. Wang, B. Cheng, L. Meng, F. Yue, W. Lan, R. Sun and J. Ren, ACS Sustain. Chem. Eng., 2019, 7, 5309-5317.

45 B. M. Trost, Science 1991, 254, 1471.

46 B. M. Trost, Acc. Chem. Res., 2002, 35, 695-705..

47 R. A. Sheldon, Green Chem., 2017, 19, 18-43.

48 K. Van Aken, L. Strekowski and L. Patiny, Beilstein J. Org. Chem., 2006, 3, 1-7.

49 S. H. Brown, C. Sjoholm, and R. M. Kelly Biotechnol. Bioeng., 1993, 41,878-886.

50 M. Moliner, Y. Román-Leshkov and M. E. Davis, PNAS, 2010, 107 (14), 6164-6168. 


\section{Supporting information}

Tuning Ca-Al-based catalysts composition to isomerize or epimerize glucose and other sugars

Maria Ventura, ${ }^{\text {a }}$ Juan A. Cecilia, ${ }^{\mathrm{b}}$ Enrique Rodríguez-Castellón, ${ }^{\mathrm{b}}$ and Marcelo E. Domine*a

a. Instituto de Tecnología Química, ITQ (UPV-CSIC). Universitat Politècnica de València. Consejo Superior de Investigaciones Científicas. Avda LoS Naranjos S/N, 46022, Valencia, Spain.

b. Departamento de Química Inorgánica, Cristalografía y Mineralogía (Unidad Asociada al ICP-CSIC), Facultad de Ciencias, Universidad de Málaga, Campus de Teatinos, 29071 Málaga, Spain.

Figure S1: FT-IR spectra of the HT-precursors samples.

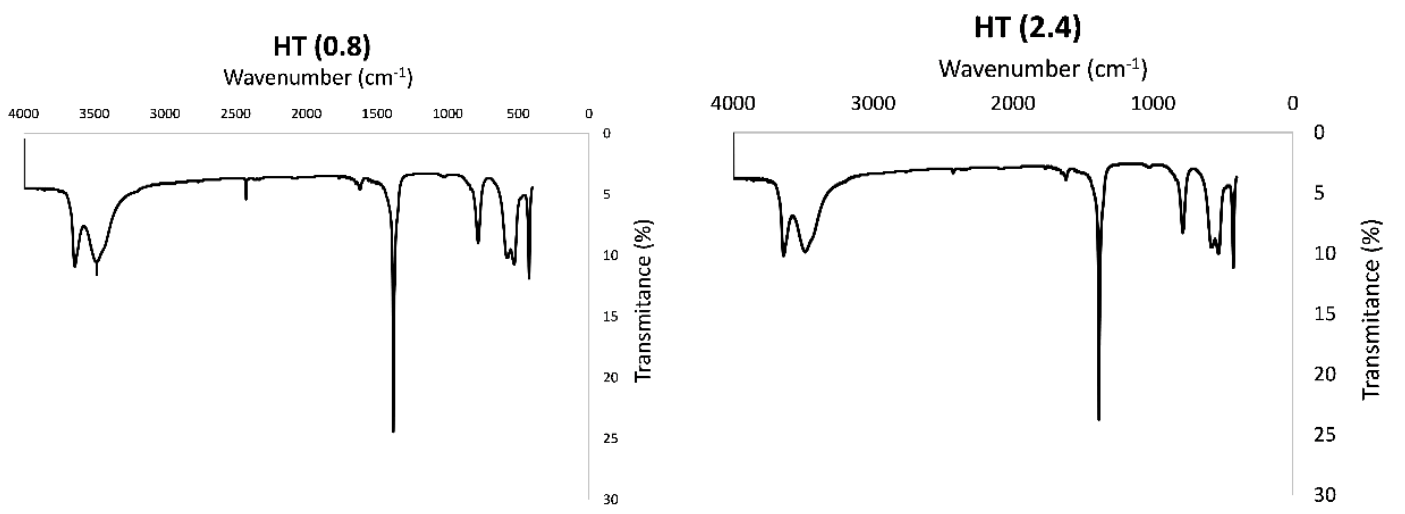

HT (3.4)

Wavenumber $\left(\mathrm{cm}^{-1}\right)$

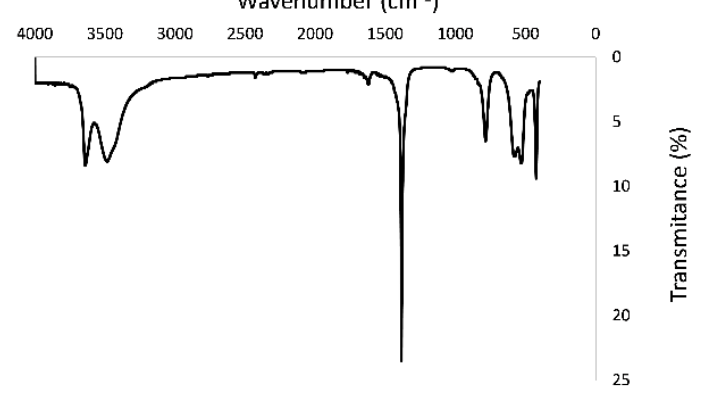


Figure S2: $\mathrm{CO}_{2}$-TPD profiles of the different $\mathrm{Ca}-\mathrm{Al}$ mixed oxides

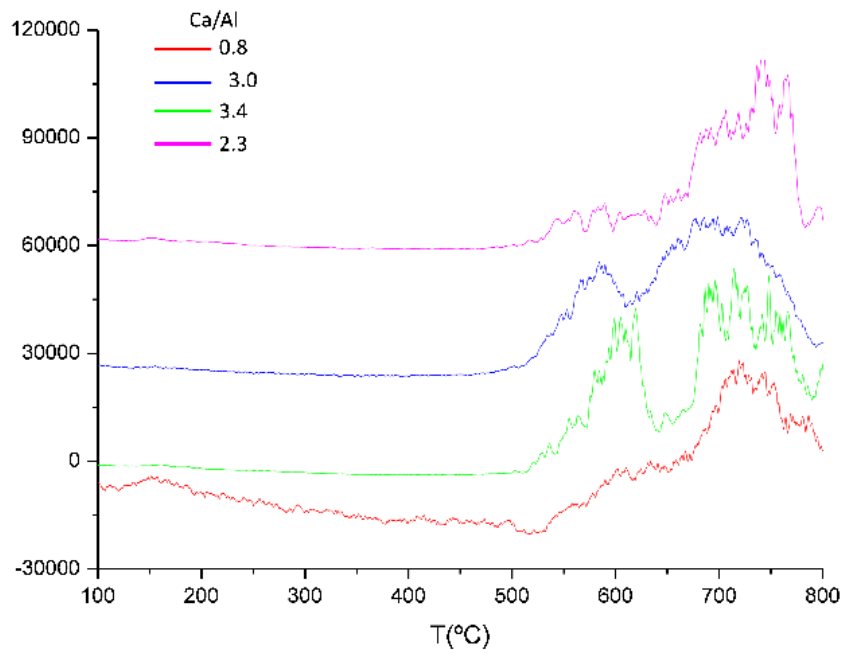

Figure S3: SEM images of HT before calcination, mixed oxides Ca-Al and SEM-EDX mapping of mixed oxides Ca-Al $(0.8 ; 2.4 ; 3.4)$, Al (red) Ca (green).

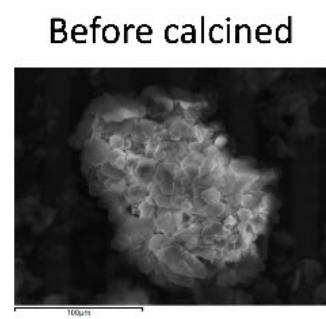

$$
\text { After calcined SEM-EDX mapping, red Ca, green Al }
$$
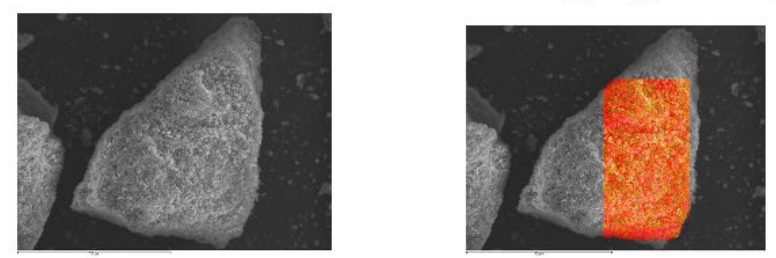

HT (0.8)

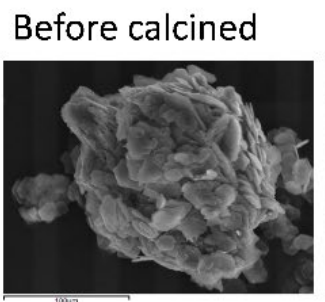

After calcined

SEM-EDX mapping green $\mathrm{Ca}$, red $\mathrm{Al}$
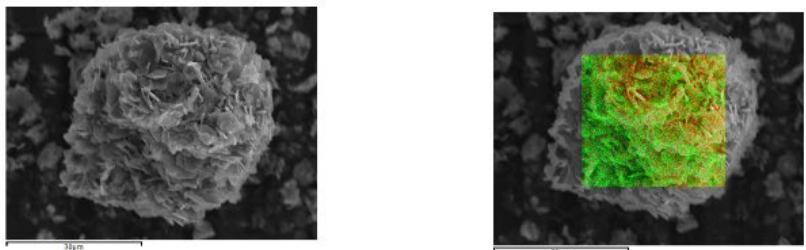

HT (2.4)

Before calcined

After calcined

SEM-EDX mapping red $\mathrm{Ca}$, green $\mathrm{Al}$
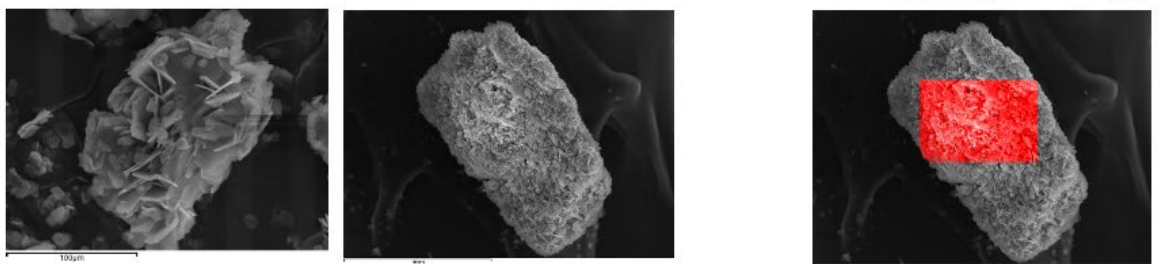

HT (3.4) 
Figure S4: Initial isomerization rate and standard deviation for each group of experiments
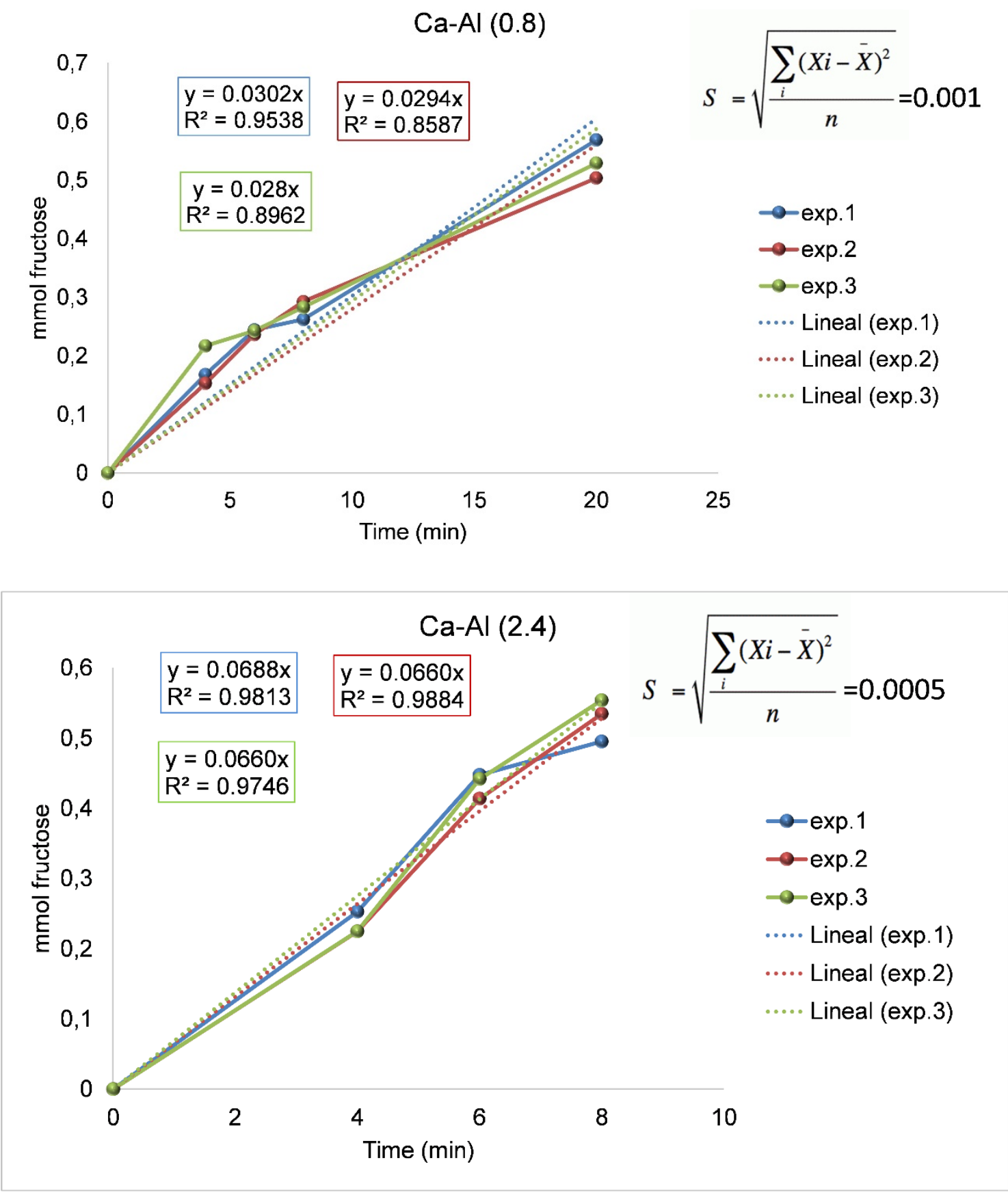

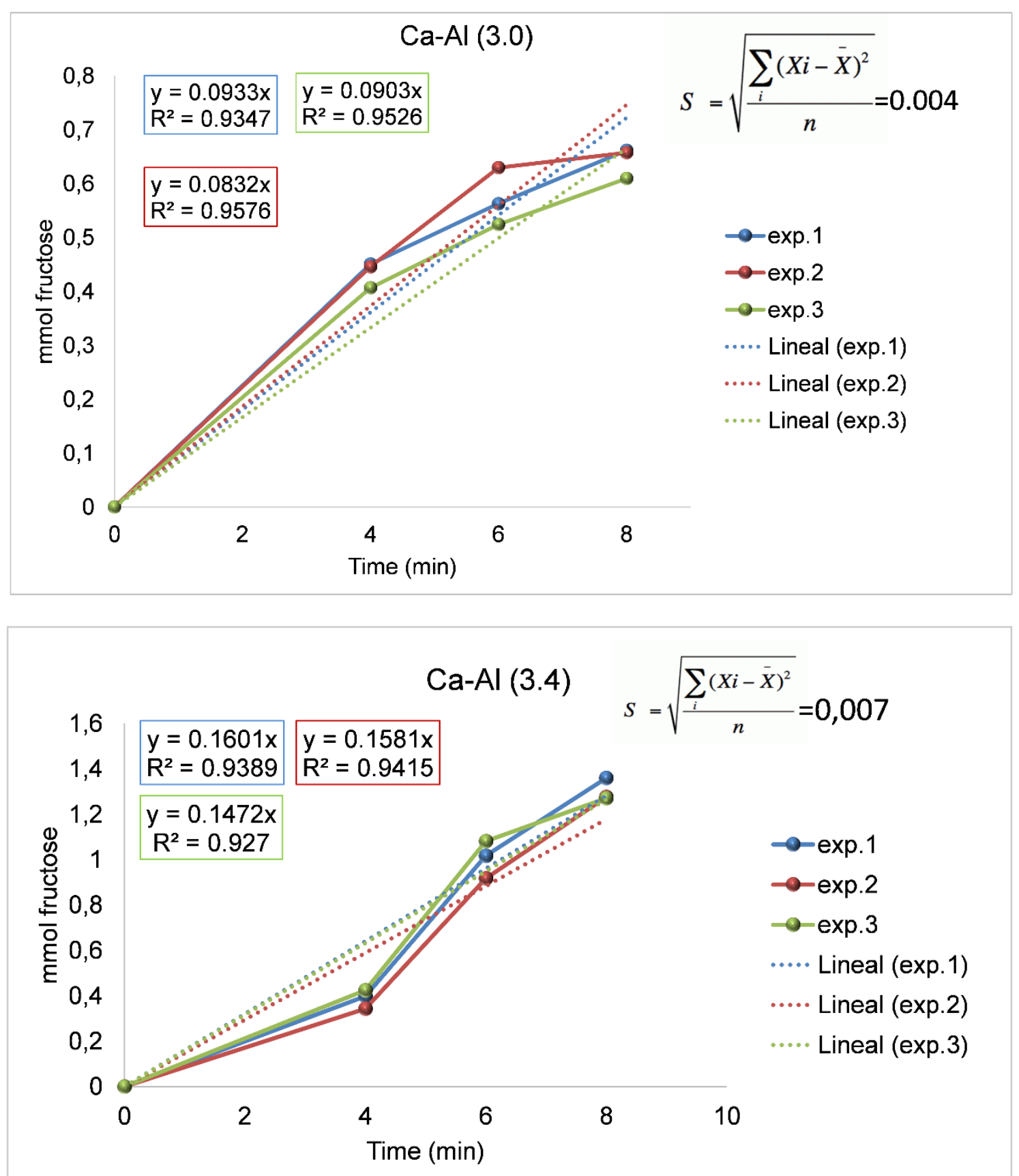
Figure S5: HPLC of the reaction mixture performed in the conditions: [Glucose] $]_{i}=0.55 \mathrm{M}, 0.05 \mathrm{~g}$ catalyst, $5 \mathrm{~mL} \mathrm{H}_{2} \mathrm{O}, \mathrm{PN}_{2}=10$ bar, $\mathrm{T}=393 \mathrm{~K}$.

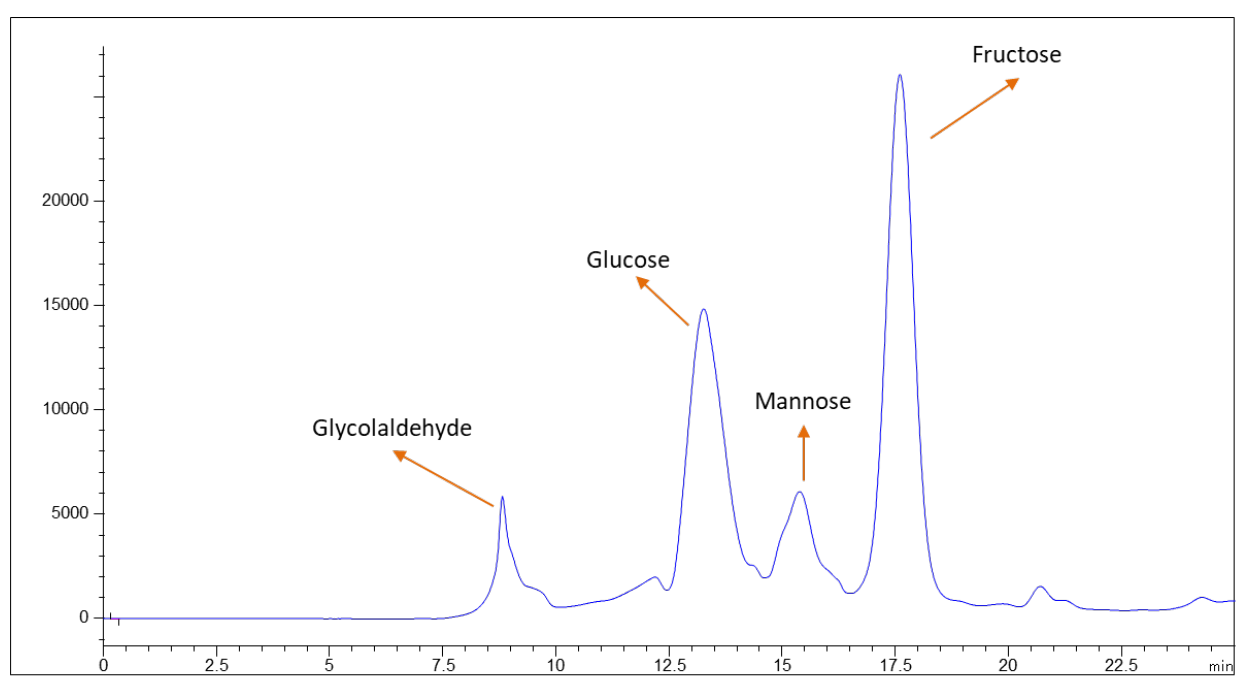

Figure S6: ${ }^{13} \mathrm{C}-\mathrm{NMR}$ of the reaction mixture, the same as above. Solvent used: mixture of $\mathrm{H}_{2} \mathrm{O}+\mathrm{D}_{2} \mathrm{O}$<smiles>O=[C]CO</smiles>

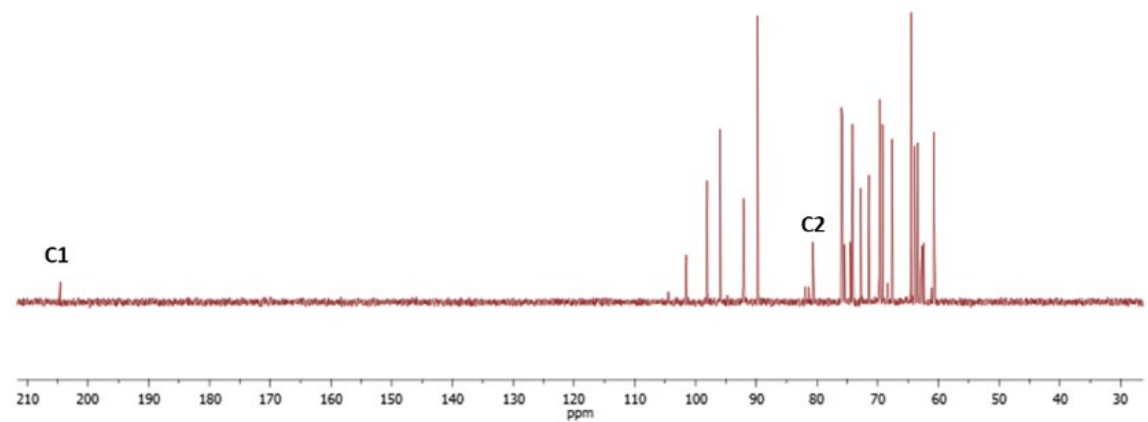

The rest of the signals are belonged to the mixture of the $\alpha$ and $\beta$ isomers of glucose + fructose. Due to the dilution grade of the products, ${ }^{1} \mathrm{H}$ NMR was not clear, and did not help to identify the mixture therefore. For this reason, it is not included. 
Figure 57: Comparison on the isomerization of fructose in absence and presence of glycolaldehyde.

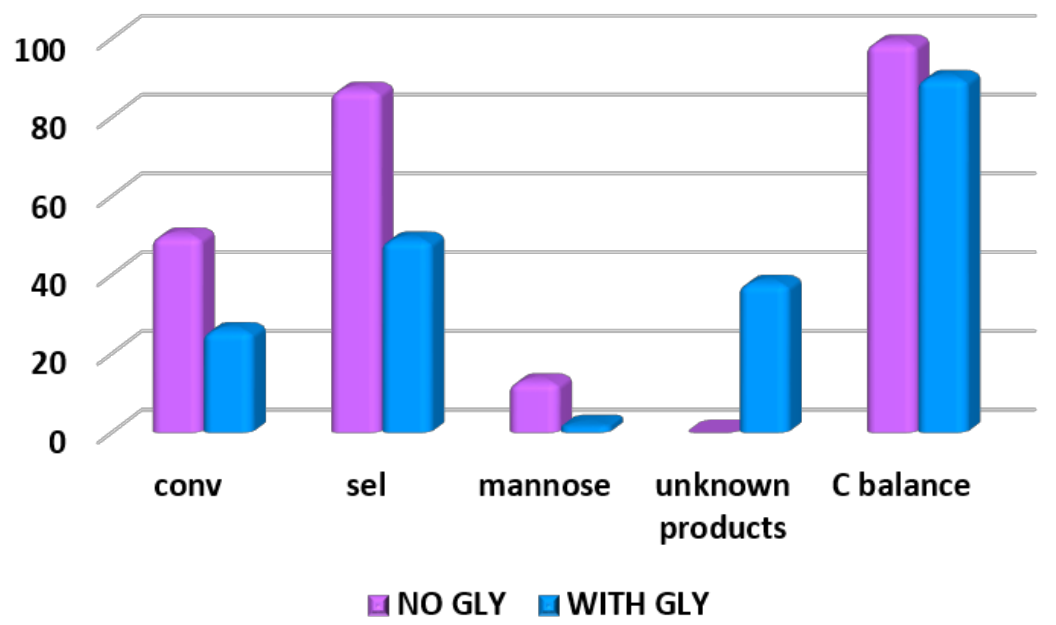

Figure S8: Overlapped HPLC of the kinetic analysis shown in Figure 7.

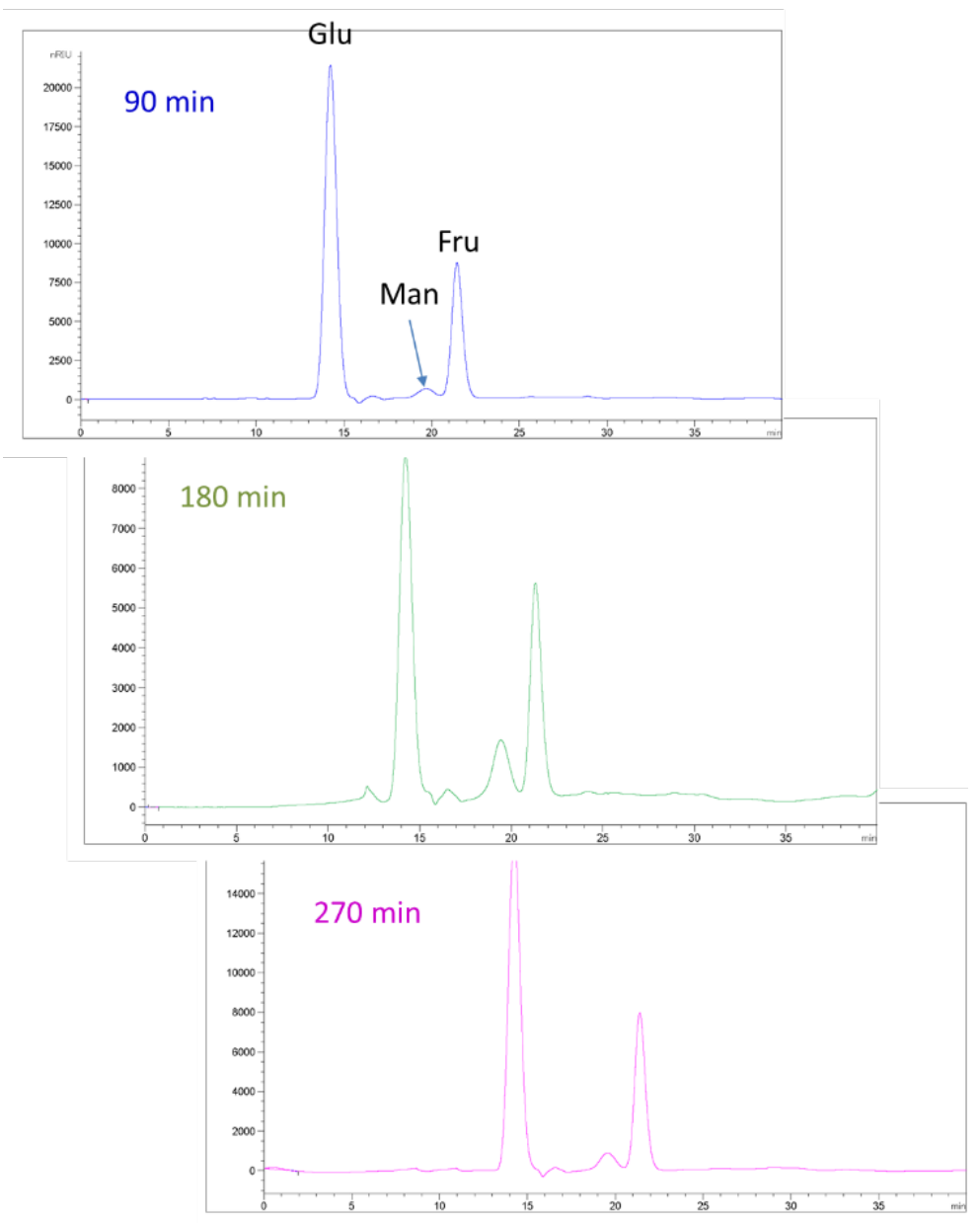


Figure S9: XRD analysis of the fresh and used catalyst for the isomerization to fructose.

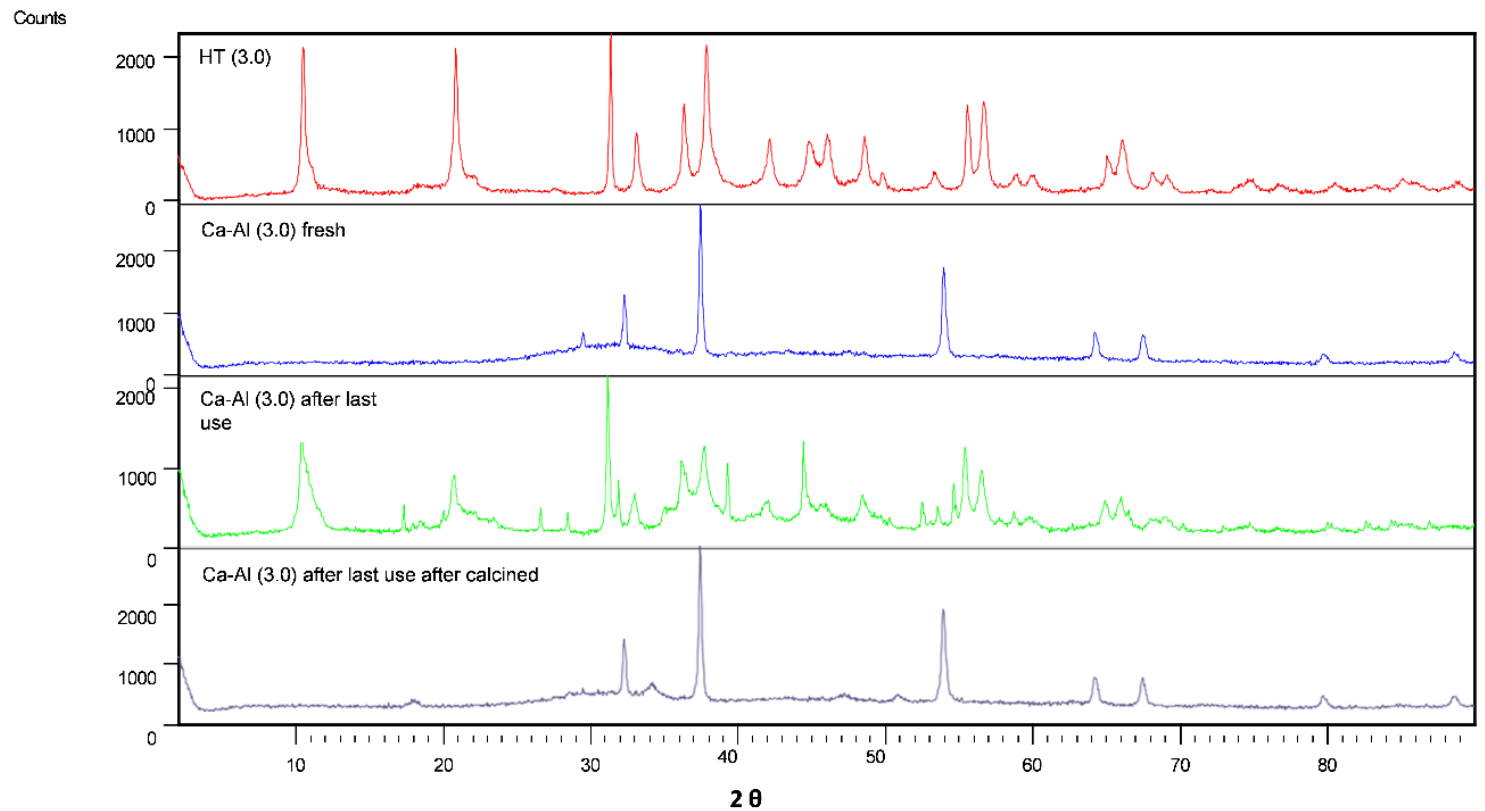

Table S1: ICP analysis of the catalysts after isomerization to fructose or epimerization to mannose.

\begin{tabular}{|c|c|c|c|c|c|c|}
\hline Entry & Reaction & run & Ca (mg) by ICP & $\mathrm{Al}(\mathrm{mg})$ by ICP & Ca loss (\%)* & Al loss (\%)* \\
\hline 1 & \multirow{3}{*}{ Isomerization } & fresh & 79.4 & 24.4 & - & - \\
\hline 2 & & $1^{\text {st }}$ & 77.4 & 24.1 & 2.5 & 1.2 \\
\hline 3 & & $5^{\text {th }}$ & 77.3 & 24 & 2.6 & 1.6 \\
\hline 4 & \multirow{3}{*}{ Epimerization } & fresh & 69.3 & 19.9 & - & - \\
\hline 5 & & $1^{\text {st }}$ & 67.3 & 19.8 & 2.8 & 0.5 \\
\hline 6 & & $5^{\text {th }}$ & 67.2 & 19.8 & 2.9 & 0.5 \\
\hline
\end{tabular}

* by comparing with reference value of fresh catalyst

\section{Green metrics calculations:}

\section{Atom Economy:}

Atom economy $=\frac{\text { Molecular weight of fructose }}{\sum \text { Molecular weight of all of the products generated in the reaction }}$

\section{E-factor:}

$$
\text { E factor }=\frac{\text { weight of undesirable products (waste) }}{\text { weight of fructose }}
$$


As it was reported by other authors [1], E-factor could be calculated considering catalysts and solvents as wastes or not considering them. For our calculations solvent was not consider because in the three cases water, the greenest and non-contaminant solvent, was used. Therefore, we have considered excluding water from these calculations. Anyway, water was considered in Ecoscale calculations (see below). However, the catalysts were considered in this E-factor since they could add toxicity to the reaction. This fact will be explained in more detail below.

Thus, E-factor for the different systems evaluated in this paper was calculated as follows:

\section{Our system}

(1) Total amount of reactants $=0.50 \mathrm{~g}$ (Glucose) $+0.05 \mathrm{~g}$ (catalyst) $=0.55 \mathrm{~g}$

(2) Amount of final product (Fructose) $=0.21 \mathrm{~g}$

Amount of waste $=(1)-(2)=0.34 \mathrm{~g}$

E-factor $=$ Amount of waste $/$ amount of products $=0.34 / 0.21=1.61$

\section{Enzymatic system}

Data from the Experimental part showed in ref. 47 (cited in the manuscript) were considered for the calculations of the E-Factor of the enzymatic system, mainly because it was impossible to found the industrial operational parameters of this process due to the trade secret of the industrial process.

(1) Total amount of reactants $=0.43 \mathrm{~g}$ (Glucose) $+0.02 \mathrm{~g}$ (enzyme) $=0.45 \mathrm{~g}$

(2) Amount of final product $=0.21 \mathrm{~g}$

Amount of waste (1) - (2) $=0.24 \mathrm{~g}$

E-factor $=1.15$

$\underline{\text { Sn- } \beta \text { system }}$

(1) Total amount of reactants $=0.50 \mathrm{~g}$ (Glucose) $+0.01 \mathrm{~g}$ (catalyst) $=0.51 \mathrm{~g}$

(2) Amount of final product $=0.12 \mathrm{~g}$ (Conversion $=30 \%$, selectivity $=80 \%$ )

Amount of waste $=0.39 \mathrm{~g}$

E-factor $=3.25$

\section{Ecoscale}

This factor was calculated by using the tools providing on internet (Webpage: http://ecoscale.cheminfo.org/calculator) free for users. Besides, the information reported by Patiny and co-workers [2] was also used. Table S2 shows the penalty points to calculate Ecoscale in general terms while Tables S3, S4 and S5 show Ecoscale calculations for our system, the enzymatic system, and the $\mathrm{Sn}-\beta$ system, respectively. It is necessary to point out that: i) the solid catalyst synthesis and ulterior activation was considered for both our catalytic 
system (Table S3) and the Sn-b system (Table S5); and ii) a final separation step via classical chromatography was included for all the evaluated systems (see Tables S3 to S5).

Table S2. Penalty points to calculate Ecoscale. Adapted with the permission of BeilsteinInstitut.

\begin{tabular}{|c|c|}
\hline Parameter & Penalty points \\
\hline 1. Yield & $(100-\%$ yield $) / 2$ \\
\hline \multicolumn{2}{|c|}{ 2. Price of reaction components (to obtain $10 \mathrm{mmol}$ of end product } \\
\hline Inexpensive $(<\$ 10)$ & 0 \\
\hline Expensive $(>\$ 10$ and $<\$ 50)$ & 3 \\
\hline Very expensive (> \$50) & 5 \\
\hline \multicolumn{2}{|l|}{ 3. Safety ${ }^{\mathrm{a}}$} \\
\hline $\mathrm{N}$ (dangerous for environment) & 5 \\
\hline $\mathrm{T}$ (toxic) & 5 \\
\hline F (highly flammable) & 5 \\
\hline E (explosive) & 10 \\
\hline $\mathrm{F}+$ (extremely flammable) & 10 \\
\hline$T+$ (extremely toxic) & 10 \\
\hline \multicolumn{2}{|l|}{ 4. Technical setup } \\
\hline Common setup & 0 \\
\hline Instruments for controlled addition of chemicals ${ }^{\mathrm{b}}$ & 1 \\
\hline Unconventional activation technique ${ }^{c}$ & 2 \\
\hline Pressure equipment, $>1 \mathrm{~atm}^{\mathrm{d}}$ & 3 \\
\hline Any additional special glassware & 1 \\
\hline (Inert) gas atmosphere & 1 \\
\hline Glove box & 3 \\
\hline \multicolumn{2}{|l|}{ 5. Temperature/time } \\
\hline Room temperature, $<1 \mathrm{~h}$ & 0 \\
\hline Room temperature, $<24 \mathrm{~h}$ & 1 \\
\hline Heating, $<1 \mathrm{~h}$ & 2 \\
\hline Heating, $>1 \mathrm{~h}$ & 3 \\
\hline Cooling to $0^{\circ} \mathrm{C}$ & 4 \\
\hline Cooling, $<0^{\circ} \mathrm{C}$ & 5 \\
\hline \multicolumn{2}{|l|}{ 6. Workup and purification } \\
\hline None & 0 \\
\hline Cooling to room temperature & 0 \\
\hline Adding solvent & 0 \\
\hline Simple filtration & 0 \\
\hline Removal of solvent with $\mathrm{bp}<150^{\circ} \mathrm{C}$ & 0 \\
\hline Crystallization and filtration & 1 \\
\hline Removal of solvent with $b p>150^{\circ} \mathrm{C}$ & 2 \\
\hline Solid phase extraction & 2 \\
\hline Distillation & 3 \\
\hline Sublimation & 3 \\
\hline Liquid-liquid extraction $^{\mathrm{e}}$ & 3 \\
\hline Classical chromatography & 10 \\
\hline
\end{tabular}

${ }^{a}$ Based on the hazard warning symbols. ${ }^{b}$ Dropping funnel, syringe pump, gas pressure regulator, etc. ${ }^{c}$ Microwave irradiation, ultrasound or photochemical activation, etc. ${ }^{d} \mathrm{ScCO}_{2}$, high pressure hydrogenation equipment, etc. elf applicable, the process includes drying of solvent with desiccant and filtration of desiccant. 
Table S3. Ecoscale for our system

\begin{tabular}{|c|c|}
\hline Parameter & Penalty points \\
\hline 1. Yield & 29 \\
\hline \multicolumn{2}{|c|}{ 2. Price of reaction components (to obtain $10 \mathrm{mmol}$ of end product) } \\
\hline Inexpensive $(<\$ 10)$ & - \\
\hline Expensive $(>\$ 10$ and $<\$ 50)$ & - \\
\hline Very expensive (>\$50) & - \\
\hline \multicolumn{2}{|l|}{ 3. Safety ${ }^{\mathrm{a}}$} \\
\hline $\mathrm{N}$ (dangerous for environment) & - \\
\hline $\mathrm{T}$ (toxic) & - \\
\hline $\mathrm{F}$ (highly flammable) & - \\
\hline E (explosive) & - \\
\hline F+ (extremely flammable) & - \\
\hline$T+$ (extremely toxic) & - \\
\hline \multicolumn{2}{|l|}{ 4. Technical setup } \\
\hline Common setup & 0 \\
\hline Instruments for controlled addition of chemicals ${ }^{\mathrm{b}}$ & 1 \\
\hline Unconventional activation technique $^{c}$ & - \\
\hline Pressure equipment, $>1 \mathrm{~atm}^{\mathrm{d}}$ & 3 \\
\hline Any additional special glassware & - \\
\hline (Inert) gas atmosphere & 1 \\
\hline Glove box & - \\
\hline \multicolumn{2}{|l|}{ 5. Temperature/time } \\
\hline Room temperature, $<1 \mathrm{~h}$ & - \\
\hline Room temperature, $<24 \mathrm{~h}$ & - \\
\hline Heating, $<1 \mathrm{~h}$ & - \\
\hline Heating, $>1 \mathrm{~h}$ & 3 \\
\hline Cooling to $0^{\circ} \mathrm{C}$ & - \\
\hline Cooling, $<0^{\circ} \mathrm{C}$ & - \\
\hline \multicolumn{2}{|l|}{ 6. Workup and purification } \\
\hline None & - \\
\hline Cooling to room temperature & - \\
\hline Adding solvent & - \\
\hline Simple filtration & - \\
\hline Removal of solvent with $\mathrm{bp}<150^{\circ} \mathrm{C}$ & 0 \\
\hline Crystallization and filtration & 1 \\
\hline Removal of solvent with $\mathrm{bp}>150^{\circ} \mathrm{C}$ & - \\
\hline Solid phase extraction & - \\
\hline Distillation & - \\
\hline Sublimation & - \\
\hline Liquid-liquid extraction $^{\mathrm{e}}$ & - \\
\hline Classical chromatography & 10 \\
\hline Total & 52 \\
\hline
\end{tabular}


Table S4. Ecoscale for the enzymatic system

\begin{tabular}{|c|c|}
\hline Parameter & Penalty points \\
\hline 1. Yield & 26 \\
\hline \multicolumn{2}{|c|}{ 2. Price of reaction components (to obtain $10 \mathrm{mmol}$ of end product) } \\
\hline Inexpensive $(<\$ 10)$ & - \\
\hline Expensive $(>\$ 10$ and $<\$ 50)$ & - \\
\hline Very expensive (>\$50) & - \\
\hline \multicolumn{2}{|l|}{ 3. Safety ${ }^{\mathrm{a}}$} \\
\hline $\mathrm{N}$ (dangerous for environment) & - \\
\hline $\mathrm{T}$ (toxic) & - \\
\hline $\mathrm{F}$ (highly flammable) & - \\
\hline E (explosive) & - \\
\hline F+ (extremely flammable) & - \\
\hline$T+$ (extremely toxic) & - \\
\hline \multicolumn{2}{|l|}{ 4. Technical setup } \\
\hline Common setup & - \\
\hline Instruments for controlled addition of chemicals ${ }^{\mathrm{b}}$ & - \\
\hline Unconventional activation technique $^{c}$ & - \\
\hline Pressure equipment, $>1 \mathrm{~atm}^{\mathrm{d}}$ & - \\
\hline Any additional special glassware & - \\
\hline (Inert) gas atmosphere & - \\
\hline Glove box & - \\
\hline \multicolumn{2}{|l|}{ 5. Temperature/time } \\
\hline Room temperature, $<1 \mathrm{~h}$ & 0 \\
\hline Room temperature, $<24 \mathrm{~h}$ & - \\
\hline Heating, $<1 \mathrm{~h}$ & - \\
\hline Heating, $>1 \mathrm{~h}$ & - \\
\hline Cooling to $0^{\circ} \mathrm{C}$ & - \\
\hline Cooling, $<0^{\circ} \mathrm{C}$ & - \\
\hline \multicolumn{2}{|l|}{ 6. Workup and purification } \\
\hline None & - \\
\hline Cooling to room temperature & - \\
\hline Adding solvent & - \\
\hline Simple filtration & - \\
\hline Removal of solvent with $\mathrm{bp}<150^{\circ} \mathrm{C}$ & - \\
\hline Crystallization and filtration & 1 \\
\hline Removal of solvent with $\mathrm{bp}>150^{\circ} \mathrm{C}$ & - \\
\hline Solid phase extraction & - \\
\hline Distillation & - \\
\hline Sublimation & - \\
\hline Liquid-liquid extraction $^{\mathrm{e}}$ & - \\
\hline Classical chromatography & 10 \\
\hline Total & 63 \\
\hline
\end{tabular}


Table S5. Ecoscale for Sn- $\beta$ system

\begin{tabular}{|c|c|}
\hline Parameter & Penalty points \\
\hline 1. Yield & 38 \\
\hline \multicolumn{2}{|c|}{ 2. Price of reaction components (to obtain $10 \mathrm{mmol}$ of end product) } \\
\hline Inexpensive $(<\$ 10)$ & 0 \\
\hline Expensive ( $>$ \$10 and $<\$ 50)$ & $(2 * 3)$ \\
\hline Very expensive (> \$50) & 5 \\
\hline \multicolumn{2}{|l|}{ 3. Safety ${ }^{a}$} \\
\hline $\mathrm{N}$ (dangerous for environment) & 5 \\
\hline $\mathrm{T}$ (toxic) & $2 * 5$ \\
\hline $\mathrm{F}$ (highly flammable) & - \\
\hline E (explosive) & - \\
\hline F+ (extremely flammable) & - \\
\hline $\mathrm{T}+$ (extremely toxic) & - \\
\hline \multicolumn{2}{|l|}{ 4. Technical setup } \\
\hline Common setup & 0 \\
\hline Instruments for controlled addition of chemicals ${ }^{\mathrm{b}}$ & 1 \\
\hline Unconventional activation technique $^{c}$ & - \\
\hline Pressure equipment, $>1 \mathrm{~atm}^{\mathrm{d}}$ & 3 \\
\hline Any additional special glassware & 1 \\
\hline (Inert) gas atmosphere & - \\
\hline Glove box & - \\
\hline \multicolumn{2}{|l|}{ 5. Temperature/time } \\
\hline Room temperature, $<1 \mathrm{~h}$ & - \\
\hline Room temperature, $<24 \mathrm{~h}$ & - \\
\hline Heating, $<1 \mathrm{~h}$ & - \\
\hline Heating, $>1 \mathrm{~h}$ & 3 \\
\hline Cooling to $0^{\circ} \mathrm{C}$ & 4 \\
\hline Cooling, $<0^{\circ} \mathrm{C}$ & - \\
\hline \multicolumn{2}{|l|}{ 6. Workup and purification } \\
\hline None & 0 \\
\hline Cooling to room temperature & 0 \\
\hline Adding solvent & 0 \\
\hline Simple filtration & 0 \\
\hline Removal of solvent with $\mathrm{bp}<150^{\circ} \mathrm{C}$ & 0 \\
\hline Crystallization and filtration & - \\
\hline Removal of solvent with $\mathrm{bp}>150^{\circ} \mathrm{C}$ & - \\
\hline Solid phase extraction & - \\
\hline Distillation & - \\
\hline Sublimation & - \\
\hline Liquid-liquid extraction $^{\mathrm{e}}$ & - \\
\hline Classical chromatography & 10 \\
\hline Total & 14 \\
\hline
\end{tabular}


Table S6: Optimization of the reaction conditions for the synthesis of HT-precursors.

\begin{tabular}{|c|c|c|c|c|c|}
\hline Entry & $\begin{array}{l}\text { Addition rate } \\
(\mathrm{mL} / \mathrm{h})\end{array}$ & $\begin{array}{l}{[\mathrm{NaOH}]} \\
(\mathrm{mol} / \mathrm{L})\end{array}$ & $\begin{array}{c}\text { [Ca] ; [Al] } \\
(\mathrm{mol} / \mathrm{L})\end{array}$ & $\begin{array}{l}\text { Ca/Al molar ratio } \\
\text { Theoretical / Measured by ICP }\end{array}$ & $\begin{array}{l}\text { Surf. Area } \\
(\mathrm{BET})\left(\mathrm{m}^{2} / \mathrm{g}\right)\end{array}$ \\
\hline 1 & 20 & 2 & \multirow{3}{*}{$1.4 ; 0.34$} & $4 / 3.1$ & 6.9 \\
\hline 2 & 20 & \multirow{4}{*}{$\begin{array}{l}2\left[\mathrm{Ca}^{2+}\right] \\
3\left[\mathrm{Al}^{3+}\right]\end{array}$} & & $3 / 3.1$ & 6.7 \\
\hline 3 & 10 & & & $3 / 2.5$ & 5.9 \\
\hline 4 & 20 & & $0.75 ; 0.25$ & $3 / 3.1$ & 5.3 \\
\hline 5 & 20 & & $2.98 ; 0.99$ & fail & - \\
\hline
\end{tabular}

Figure S10: Comparison on XRD of entry 1 (red), 2 (blue)

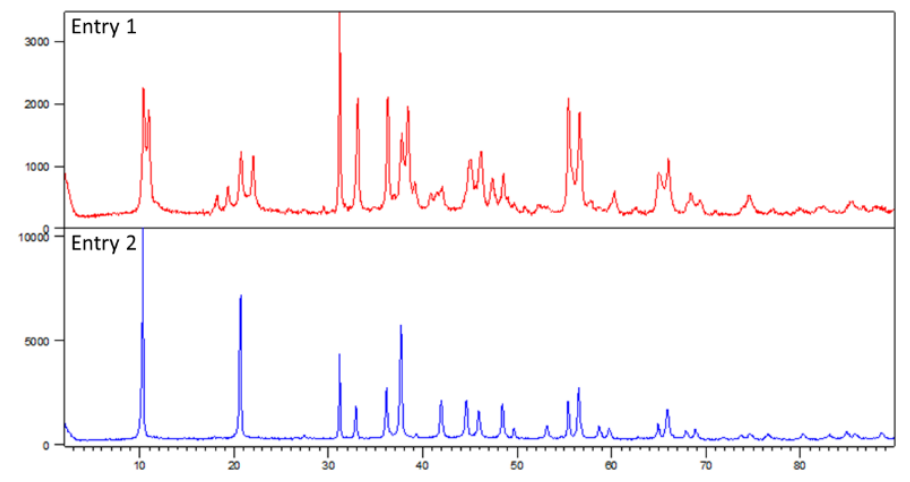

Figure S11: Comparison on XRD of entry 3 (green), 2 (blue)

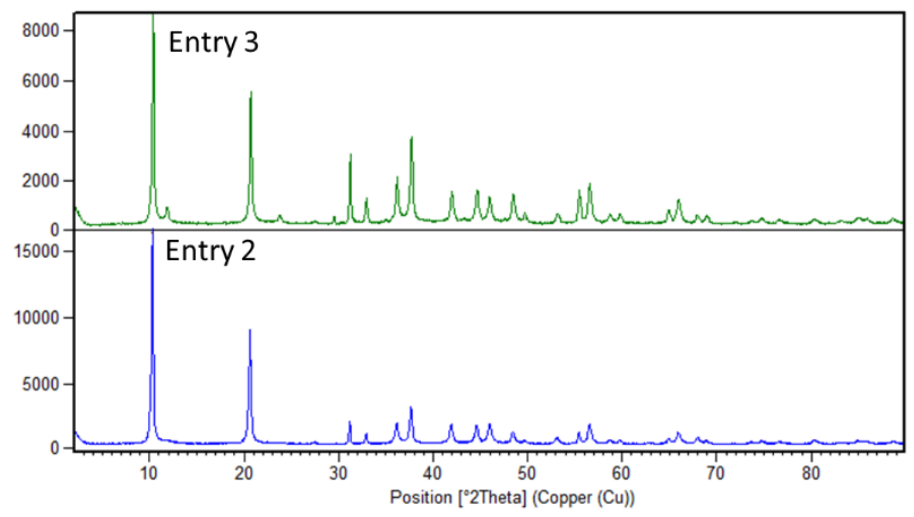


Figure S12: Comparison of entry 4 (pink), 2 (blue).

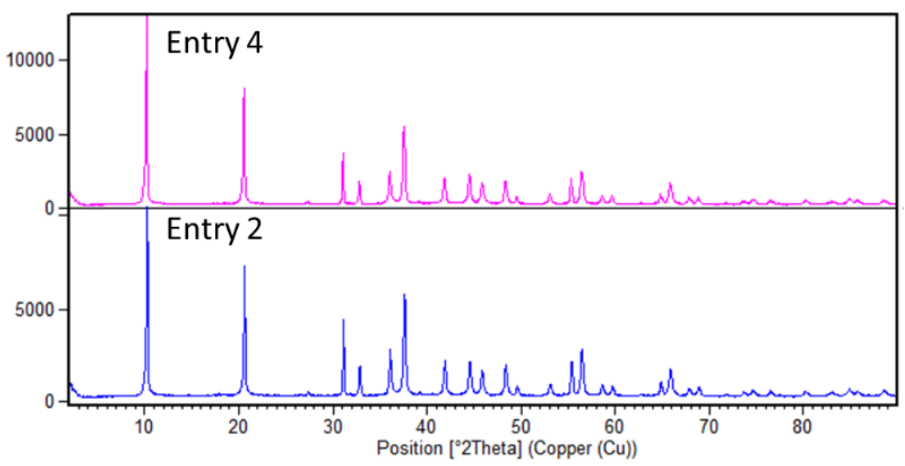

Figure S13: Comparison of entry 5 (grey), 2 (blue).

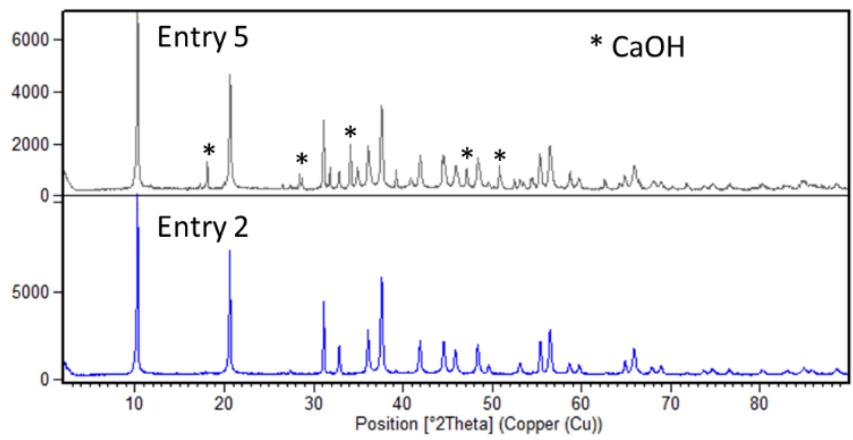

\section{Kinetics of Glucose isomerization and epimerization reactions}

Catalytic results showed in Figures 7 and 9 of the manuscript were obtained from reactions repeated several times, as it is shown in Figures S14 and S15. The data accuracy was checked being within normal error limits, taking into account experimental errors such as HPLC measurements and experimental proceeds. 


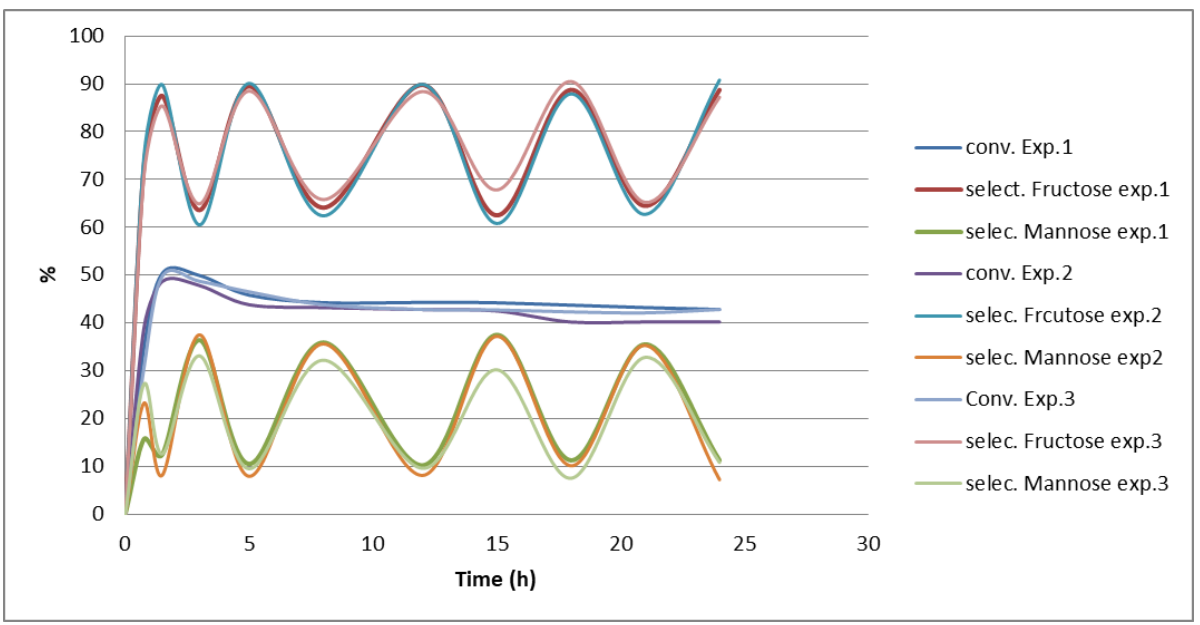

Figure S14. Kinetics of the isomerization of glucose using Ca-Al (3) as catalyst.

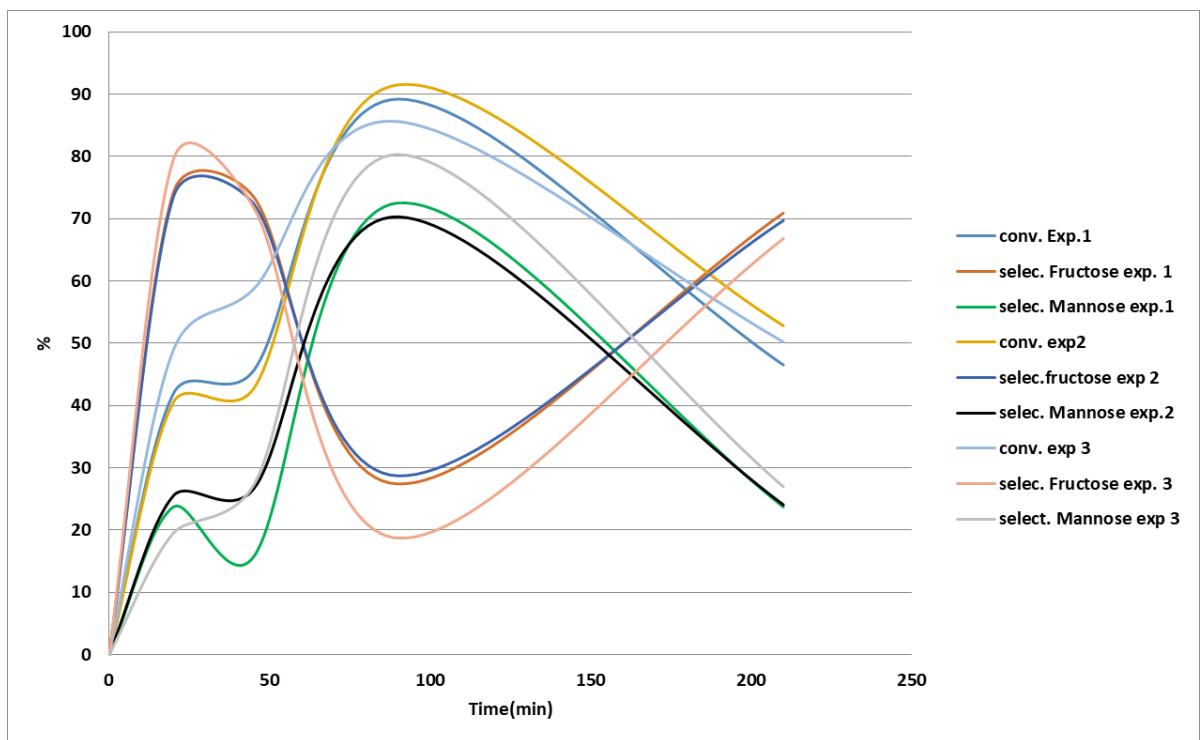

Figure S15. Kinetics of the epimerization of glucose using Ca-Al (3.4) as catalyst.

Rate constants $\mathrm{k} 1$ for the isomerization to fructose and $\mathrm{k} 2$ for the epimerization to mannose were calculated from the slope of the graphic in Figure S14 and S15, respectively, where the reaction is in the equilibrium, obtaining the following values: $k 1=0.020 \mathrm{~min}^{-1}$ and $k 2=0.021$ $\min ^{-1}$, Figures S16 and S17. 


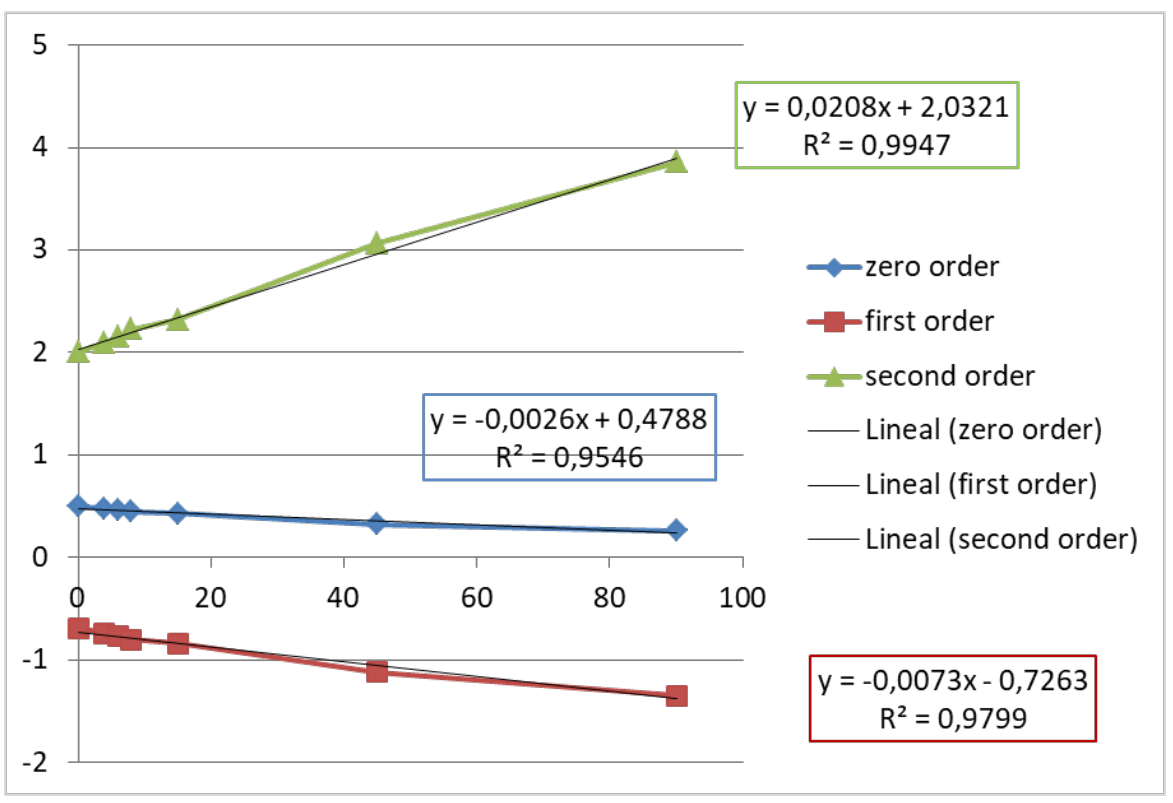

Figure S16. Calculations performed to elucidate the kinetic model for isomerization of glucose to fructose.

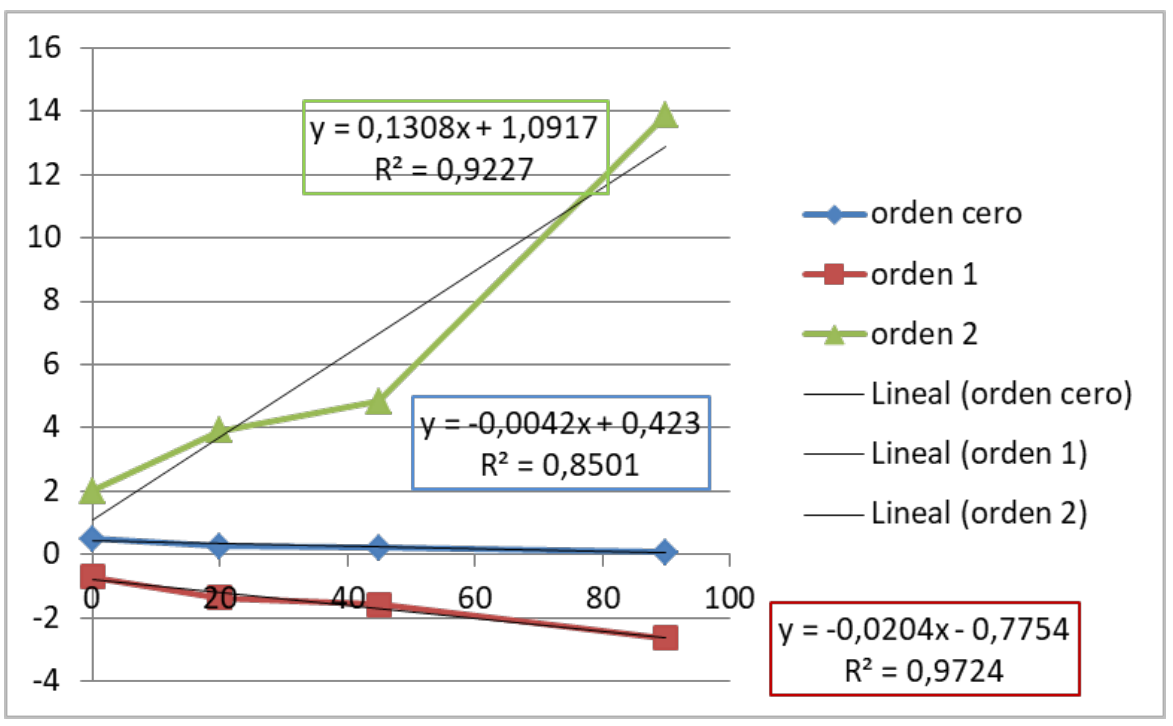

Figure S17. Calculations performed to elucidate the kinetic model for epimerization of glucose to mannose.

Although in the first case (Fig. S16), apparently the data adjust for both first-order $\left(R^{2}=0.9979\right)$ and second-order $\left(R^{2}=0.9947\right)$ models, the first-order model is the more plausible for a heterogeneous catalyst mechanism and it was preferred for the glucose-to-fructose isomerization. In the case of the epimerization to mannose, a first-order model adjusts pretty well with the data (Fig. S17). The equations proposed for both reactions rates are the following:

Isomerization to fructose: $\quad \mathrm{v}=k[$ Glucose $]$

Epimerization to mannose: $\quad \mathrm{v}=k[$ Glucose $]$ 
In this sense, and taking into account that both constants have practically the same value, this could be more consistent with the kinetic model of Competitive First Order Reactions, where the equations for fructose and mannose (calculated by a well-known mathematical model) are the following:

$$
[B]=\left[k_{1}[A]_{0}-\left(k_{1}+k_{2}\right)-\left(k_{1}+k_{2}\right)_{t}\right]_{t} ;[C]=k_{2}[A]_{0} k_{1}+k_{2}\left[1-e^{-(k 1+k 2) t}\right]
$$

Where $\mathrm{A}=$ glucose; $\mathrm{B}=$ fructose; and $\mathrm{C}=$ mannose.

\section{Different calcination temperatures}

Figure S18: Correlation between calcination temperature, glucose conversion and fructose selectivity.

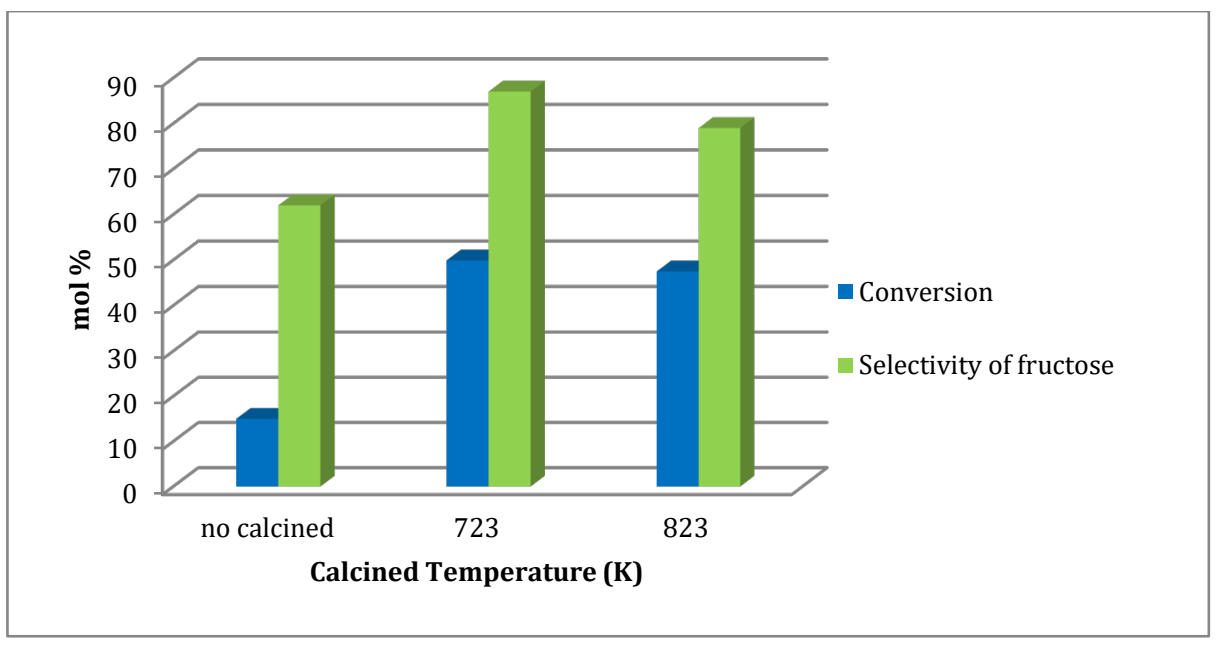

As synthesized Ca-Al hydrotalcite-type materials (without calcination) possess a poor reactivity in the isomerization reaction because they present the layered structure of double metallic hydroxides typical of hydrotalcite-type materials, where water and nitrate anions from metal precursors are still present in the interlayer system, thus reducing basicity and activity of the catalysts. Once the hydrotalcite-precursor is calcined (at $723 \mathrm{~K}$ during $4 \mathrm{~h}$, see Experimental), interlayered water and precursors moieties are eliminated and the corresponding Ca-Al mixed oxide structure is obtained, as it is confirmed by XRD measurements of the solids before and after calcination (see Fig. 1 in the manuscript). The temperature and methodology (from room temperature until $723 \mathrm{~K}$ with a temperature rate of $2{ }^{\circ} \mathrm{C} / \mathrm{min}$ under air atmosphere, and then kept at $723 \mathrm{~K}$ during $4 \mathrm{~h}$ under $\mathrm{N}_{2}$ atmosphere) followed for calcination treatment were selected based on previous studies performed in our Institution (ITQ, UPV - CSIC) by working with different Metal-Al mixed oxides derived from hydrotalcite-type materials. [3, 4] In this sense, the activity of the more active $\mathrm{Ca}-\mathrm{Al}$-based catalyst $(\mathrm{Ca} / \mathrm{Al}$ molar ratio $=3)$, synthesized with two different calcination temperatures (723 and $823 \mathrm{~K}$ ), were investigated. Figure S18 shows the correlation between calcination temperature, glucose conversion, and selectivity to fructose. As can be seen, best results were attained when the Ca-Al material was calcined at $723 \mathrm{~K}$. No great differences in glucose conversion were observed when the calcination temperature was 
increased up to $823 \mathrm{~K}$, just a small decrease in selectivity to fructose. However, the Ca-Al material without calcination showed much lower activity (in terms of both glucose conversion and selectivity to fructose) than the calcined ones. Therefore, the Ca-Al-based catalyst calcined at $723 \mathrm{~K}$ (selected temperature) was chosen as the most active catalyst.

\section{Mechanism by NMR}

The reaction using glucose-2- $d_{1}$ as substrate was performed in the same conditions as those described for the isomerization of glucose to fructose with unlabeled glucose. We could elucidate the rate-determining step in the glucose isomerization due to the decrease in the isomerization rate when using glucose-2-d1. This indicates the $\mathrm{C} 2-\mathrm{H}$ bond cleavage as the ratedetermining step. Table $\mathrm{S} 7$ shows a comparison between the two reactions.

Table S7. Isotope effect on the isomerization of glucose.

\begin{tabular}{|c|c|c|}
\hline Substrate & Conversion (\%) & Fructose yield (\%) \\
\hline Glucose & 50 & 43 \\
\hline Glucose-2- $d_{1}$ & 25 & 18 \\
\hline
\end{tabular}

Reaction conditions: [Glucose] $=0.55 \mathrm{M}, 0.05 \mathrm{~g}$ catalyst, $5 \mathrm{~mL} \mathrm{D} \mathrm{D}_{2} \mathrm{O}, \mathrm{PN}_{2}=10$ bar, temperature $=$ $363 \mathrm{~K}$, reaction time $=90 \mathrm{~min}$.

More investigations were performed to clarify the mechanism by NMR spectroscopy. Due to the reaction was done in $\mathrm{D}_{2} \mathrm{O}$, the low concentration of the compounds hindered to follow the reaction by ${ }^{1} \mathrm{H}-\mathrm{NMR}$; therefore, it was followed by ${ }^{13} \mathrm{C}-\mathrm{NMR}$. If the mechanism goes via LdB-AvE, the common mechanism for base-catalyzed isomerization, the products synthesized from glucose-2- $d_{1}$ could lack a deuterium atom in their structure, therefore $C 1$, called $a, b$ in $\alpha$ fructofuranose and $c, d$ in $\beta$-fructofuranose, Figure S19, would be classified by ${ }^{13} C$ and DEPT-135 as secondary carbon. However, when the reaction was performed and following by NMR, this $a$, $b$ was classified as tertiary carbon and give positive peaks in DEPT-135 and $c, d$ as secondary carbon giving negative peaks. Under these results seems that both mechanisms, basic and acidcatalyzed are got involved. The reaction was repeated several times giving the same ${ }^{13} \mathrm{C}$ spectrums. In Figure S20 is showed LdB-AvE mechanism while Figure S21 shows isomerization catalyzed by Lewis acid catalysts. Although the first mechanism is the more plausible for our catalytic system, the NMR studies performed are not conclusive. 


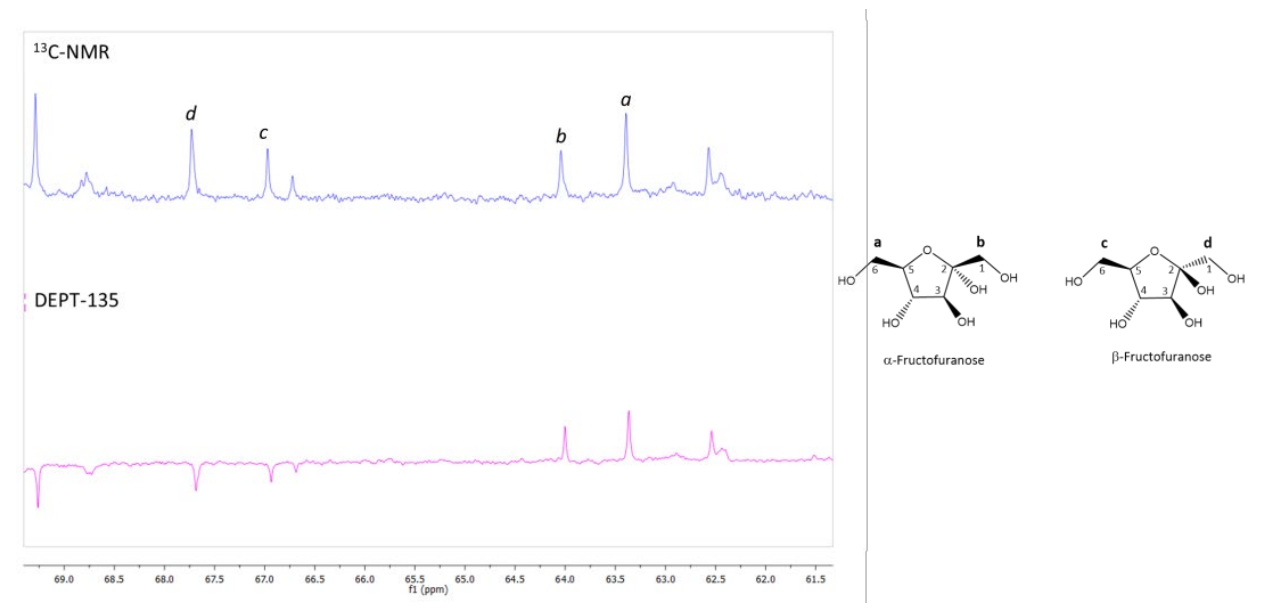

Figure S19. ${ }^{13} \mathrm{C}$-NMR and DEPT-135 spectra in the range of $61-69$ for the reaction mixture obtained from glucose-2- $d_{1}$<smiles>O=CCOC[C@@H](O)[C@@H](O)[C@@H](O)[C@H](O)[C@@H](O)C=O</smiles>

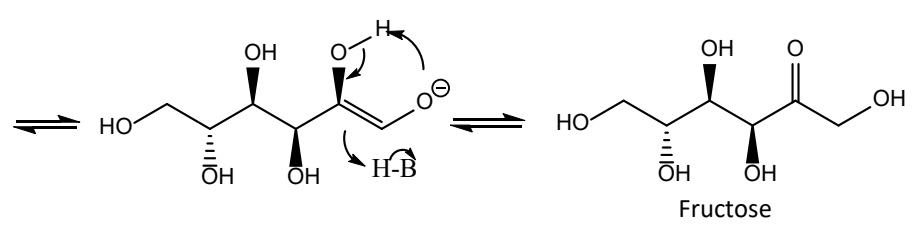

Figure S20. Isomerization of Fructose via LdB-AvE mechanism catalyzed by basic catalysts (B).

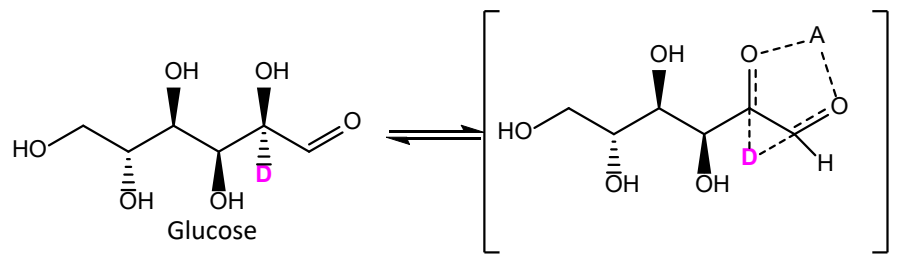<smiles>[2H]C(O)C(=O)[C@@H](O)[C@@H](O)[C@H](O)COC#C</smiles>

Figure S21. Isomerization of Glucose to fructose via intramolecular 1,2-hydride shift catalyzed by Lewis acids (A). 


\section{Additional Explanation for Fructose/Mannose Selectivity}

Catalytic results showed in Figures 7 and 9 of the manuscript were obtained from reactions repeated several times (see Figures S14 and S15 above). The data accuracy was checked carefully, and we are confident that these data are not arising from either the error or the coincidence. Therefore, the experimental evidences suggest an oscillating system in this case. In this sense, there are many oscillating chemical reactions reported in literature closely related to glucose isomerization [5]. In this reference, the author explain some oscillating reactions, providing mechanism and examples. Even if isomerization of glucose to fructose does not appear, the glycolytic pathway via enzymatic system is described as an example of oscillating reactions [5], which is related to glucose isomerization.

In addition to that, some key points of our research and recent reports that could support our experimental results are discussed in the next paragraphs.

On one hand, Y. Wang et al. [6] have studied nitrogen-doped mesoporous carbon materials as catalysts for the isomerization of glucose to fructose. They did not observe an oscillating reaction but after a certain reaction time, a decrease in the fructose yield is observed. As the reaction was not kept during more time, it was not possible to observe a new increase in the fructose yield; but does not mean that it could not occur. At the same time, the authors affirm "literally" that "the maximum yield of fructose varying with temperature verify that isomerization reaction of glucose to fructose is an endothermic reversible reaction, which was consistent with the literature" $[7,8]$ and that "basicity cannot only promote the direct glucose isomerization into fructose, but also the further glucose or fructose transformation". In this sense, two facts are connected with our observations:

i) The isomerization is a reversible reaction, which depends on temperature. As matter of fact, we did not observe any oscillation when experiments were performed at lower temperature or at atmospheric pressure. And,

ii) The high basicity of our catalysts could promote the transformation of fructose to glucose, as we have observed when fructose is getting at substrate; glucose is obtained as the major reaction product (See Table 5 in the manuscript).

Moreover, R. Otomo et al. [9] have observed the same decrease in fructose yield with the increased reaction time. The authors explained this decrease because of fructose degradation but without providing any evidence supported by adequate analysis; therefore, the possibility of mannose formation is opened.

On the other hand, there is other fact to consider that is the analysis procedure of the reaction mixture. To our knowledge, the proper HPLC-type column allowing detecting the entire product isomers derived from glucose is an $8 \%$ cross-linked resin- $\mathrm{Ca}$ (or $\mathrm{Pb}$ ) ionic form. Conversely, the authors in the above-mentioned papers use different columns, such as Welch Ultimate $\mathrm{XB}-\mathrm{NH}_{2}$ and ROA-Organic acids, respectively. These HPLC columns are not able to give high resolution to separate and detect the mixture of glucose, fructose and mannose. Therefore, errors may exist in the analysis of the reaction mixture that prevents the detection of all of the components present in the mixture.

Another point that we would like to clarify refers to the reaction conditions here employed. In our case, we performs experiments at 10 bar of $\mathrm{N}_{2}$ pressure. Classically, the isomerization of glucose is performed at atmospheric pressure, as many works confirm. The equilibrium constant for the reaction is calculated at that pressure; thus, pressure increasing in an equilibrium type 
system will displace the reaction to the formation of products, in our case fructose, reaching another equilibrium state.

Finally, it is important to emphasize that the oscillating system was only evidenced when the glucose isomerization reaction was done in a batch reactor at $393 \mathrm{~K}$ and 10 bars of pressure during longer times, this allowing us to observe the variation between fructose and mannose selectivity, while glucose conversion remains practically constant (see Fig. 7 in the manuscript). On the contrary, when the experiment was carried out at lower temperatures $(<363 \mathrm{~K})$ and at atmospheric pressure only fructose was observed as the main product. More importantly, experiments carried out at shorter contact times in a continuous flow fixed-bed catalytic reactor (at $>363 \mathrm{~K}$ and atmospheric pressure) over our Ca-Al-type catalysts mainly gives fructose as product with excellent selectivity [9].

\section{References}

[1] I. Serra, S. Daly, A.R. Alcantara, D. Bianchi, M. Terreni, D. Ubiali, RSC Adv., 5 (2015) 2356923577.

[2] K. Van Aken, L. Strekowski, L. Patiny, Beilstein J. Org. Chem., 2 (2006) 3-3.

[3] M.J. Climent, A. Corma, P. De Frutos, S. Iborra, M. Noy, A. Velty, P. Concepción, J. Catal., 269 (2010) 140-149.

[4] A. Corma, S.B. Abd Hamid, S. Iborra, A. Velty, J. Catal., 234(2) (2005) 340-347.

[5] Joseph Higgins in "The theory of oscillating reactions", Industrial and Engineering Chemistry, Vol. 59, 5, 1967.

[6] Y. Wang, J. Wang, Y. Zhang, F. Song, Y. Xie, M. Wang, H. Cui and W. Yi, Catal. Lett., (2019) (Doi: 10.1007/s10562-019-03020-1).

[7] I. Graça, M.C. Bacariza, A. Fernandes et al., Appl. Catal. B: Environ., 224 (2018) 660-670.

[8] M.D. Mello and M. Tsapatsis, ChemCatChem, 10 (2018) 2417-2423.

[9] R. Otomo, M. Fujimoto, M. Nagao and Y. Kamiya, Mol. Catal., 475 (2019) 110479-110487.

[10] M. Ventura, J. Mazarío and M.E. Domine. "Isomerization of glucose-to-fructose in a continuous flow reactor using Ca-Al mixed oxide as heterogeneous catalyst". Manuscript in preparation. 